\title{
Prediction of resistance to chemotherapy in ovarian cancer: a systematic review
}

\author{
Katherine L Lloyd ${ }^{1 *}$, lan A Cree ${ }^{2}$ and Richard S Savage ${ }^{2,3}$
}

\begin{abstract}
Background: Patient response to chemotherapy for ovarian cancer is extremely heterogeneous and there are currently no tools to aid the prediction of sensitivity or resistance to chemotherapy and allow treatment stratification. Such a tool could greatly improve patient survival by identifying the most appropriate treatment on a patient-specific basis.

Methods: PubMed was searched for studies predicting response or resistance to chemotherapy using gene expression measurements of human tissue in ovarian cancer.

Results: 42 studies were identified and both the data collection and modelling methods were compared. The majority of studies utilised fresh-frozen or formalin-fixed paraffin-embedded tissue. Modelling techniques varied, the most popular being Cox proportional hazards regression and hierarchical clustering which were used by 17 and 11 studies respectively. The gene signatures identified by the various studies were not consistent, with very few genes being identified by more than two studies. Patient cohorts were often noted to be heterogeneous with respect to chemotherapy treatment undergone by patients.

Conclusions: A clinically applicable gene signature capable of predicting patient response to chemotherapy has not yet been identified. Research into a predictive, as opposed to prognostic, model could be highly beneficial and aid the identification of the most suitable treatment for patients.
\end{abstract}

Keywords: Ovarian cancer, Chemoresistance, Predictive model, Statistical modelling

\section{Background}

Ovarian cancer is the fifth most common cancer in women in the UK and accounted for $4 \%$ of cancer diagnoses in women between 2008 and 2010 [1]. Worryingly, it was also responsible for $6 \%$ of cancer-related deaths in women over the same time period [1] and the fiveyear survival of women diagnosed with ovarian cancer between 2005 and 2009 was $42 \%$ [2]. It has been observed that although $40 \%-60 \%$ of patients achieve complete clinical response to first-line chemotherapy treatment [3], around $50 \%$ of these patients relapse within 5 years [4] and only $10 \%-15 \%$ of patients presenting with advanced stage disease achieve long-term remission [5]. It is thought that the high relapse rate is at least in part due to resistance to chemotherapy, which may be inherent or acquired by altered gene expression [6].

\footnotetext{
${ }^{*}$ Correspondence: K.Lloyd.1@warwick.ac.uk

1 MOAC DTC, University of Warwick, Gibbet Hill Road, CV4 7AL, Coventry, UK

Full list of author information is available at the end of the article
}

For ovarian cancer in the UK, the standard of care for first-line chemotherapy treatment recommended by the National Institute for Health and Care Excellence is 'paclitaxel in combination with a platinum-based compound or platinum-based therapy alone' [7]. This uniform approach ignores the complexity of ovarian cancer histologic types, particularly as there is evidence to suggest differences in response [8]. Winter et al. [9] investigated the survival of patients following paclitaxel and platinum chemotherapy and found histology to be a significant predictor of overall survival in multivariate Cox proportional hazards regression.

Improvement in survival has also been poor in ovarian cancer. Between 1971 and 2007 there was a 38\% increase in relative 10-year survival in breast cancer, whereas the increase in ovarian cancer was $17 \%$ [10]. This difference in progress is likely to be due, at least in part, to the lack of tools with which to predict chemotherapy response in ovarian cancer. 
Gene expression based tools for the prediction of patient prognosis after surgery or chemotherapy are currently available for some cancers. For example, MammaPrint uses the expression of 70 genes to predict the likelihood of metastasis in breast cancer [11]. Similarly, the Oncotype $\mathrm{DX}^{\circ}$ assay uses the expression of a panel of 21 genes to predict recurrence after treatment of breast cancer [12]. The Oncotype DX assay is also available for colon [13] and prostate cancers [14]. The development of a similar tool for ovarian cancer could greatly improve patient prognosis and quality of life by guiding chemotherapy choices. The prediction of cancer prognosis using gene signatures is a popular research field, within which a wide variety of approaches have been considered. Popular RNA or protein expression measurement techniques include cDNA hybridisation microarrays, endpoint and quantitative reverse transcription PCR, and immunohistochemistry approaches.

Another variable aspect of studies predicting chemotherapy response is the computational and statistical approaches utilised. One of most popular methods for survival analysis is Cox proportional hazards regression. This model assumes that the hazard of death is proportional to the exponential of a linear predictor formed of the explanatory variables. This model has the advantage that, unlike many other regression techniques, it can appropriately deal with right-censored data such as that found in medical studies where patients leave before the end of the study period [15].

Other popular modelling techniques include linear models, support vector machines, hierarchical clustering, principal components analysis and the formation of a scoring algorithm. When dealing with data sets of varying sizes it is important to consider the number of samples and the amount of data per patient when choosing a modelling method. If the number of patients is large it is clear that a model will be better informed about the population from which the patient sample was drawn, and hence is likely to generalise more effectively to independent data sets. As the number of measurements per patient increases, the dimensionality and hence the flexibility of the model may increase. However, it is also important that the number of patients is sufficiently large to supply enough information about the factors being considered. Of the models identified here, linear models are relatively restrictive as the relationship between any factor and the outcome is assumed to be linear and so are suitable for smaller data sets. Conversely, hierarchical clustering simply finds groups of similar samples and there are minimal assumptions concerning the relationship between factors and outcome.

Classification models are used to predict which of a number of groups an individual falls into and are used for categorical variables, such as tumour grade and having or not having a disease. For visualisation and the assessment of classification model predictive power, a Kaplan-Meier plot is often combined with the log-rank test to investigate significance. It is worth noting that this method does not compare predictions with measurements, it simply considers the difference in survival between groups.

Many of the studies identified by this review involved developing a model using one set of samples, a training set, followed by testing of the model carried out on an independent set of samples, the test or validation set. This partitioning of samples is important as it allows the generalisability of the model to be assessed, and hence guards against over-fitting. If this check is not carried out, the true predictive ability of the model will not be known.

The aim of this review is to investigate the literature surrounding the prediction of chemotherapy response in ovarian cancer using gene expression. It has been observed, for example by Gillet et al. [16], that gene signatures obtained from cancer cell lines are not always relevant to in vivo studies, and that cell lines are inaccurate models of chemosensitivity [17]. The search was therefore restricted to studies involving human tissue in order to ensure that the resulting gene signatures are applicable in a clinical setting. It was also specified that the study must involve patients who have undergone chemotherapy treatment, so that the effects of resistance may be investigated.

\section{Methods}

\section{Search methodology}

The aim of this review is to investigate the literature on the prediction of chemoresistance in patients with ovarian cancer. Therefore, the six most important requirements identified were:

- Concerned with (specifically) ovarian cancer

- Patients were treated with chemotherapy

- Gene expression was measured for use in predictions

- Predictions are related to a measure of chemoresistance (e.g. response rates, progression-free survival)

- Measurements were taken on human tissue (not cell lines)

- The research aim is to develop a diagnostic tool or predict response

A PubMed search was carried out on 6th August 2014 to identify studies fulfilling the above requirements. The search terms may be found in Additional file 1. This search resulted in 78 papers.

\section{Filtering}

The search results were filtered twice, once based on abstracts and once based on full texts, by KL. An overview 
of the filtering process may be found in Figure 1. For the abstract-based filtering, papers were excluded if the six essential criteria were not all met, if the paper was a review article or if the paper was non-English language. This resulted in 48 papers remaining. For the full-text-based filtering, exclusion was due to not fulfilling the search criteria or papers that were not available. 42 papers were remaining after full-text-based filtering.

\section{Data extraction}

Data was extracted using a pre-defined table created for the purpose. Extraction was carried out in duplicate by a single author $(\mathrm{KL})$ with a wash-out period of 3 months to avoid bias. Variables extracted were: author, year, journal, number of samples, number of genes measured, study end-point, tissue source, percentage cancerous tissue, gene or protein expression measurement technique, sample histological types and stages, patient prior chemotherapy, modelling techniques applied, whether the model accounts for heterogeneity in patient chemotherapy, whether the model was prognostic or predictive, whether the model was validated, model predictive ability including any metrics or statistics, and the genes found to be predictive.

\section{Bias analysis}

Bias in the studies selected for the systematic review was assessed according to QUADAS-2 [18], a tool for the quality assessment of diagnostic accuracy studies. Levels of evidence were also assessed according to the CEBM 2011 Levels of Evidence [19]. Results of these analyses may be found in Additional files 2 and 3. Briefly, the majority of studies were considered to be low risk, with six studies judged to have unclear risk for at least one domain and seven studies judged to be high risk for at least one domain. Thirty-six studies where judged to have evidence of level 2, with the remaining six having evidence of level 3. These levels of risk and evidence suggest that the majority of conclusions drawn from these studies are representative and applicable to the review question.

\section{Gene set enrichment}

Gene set enrichment analysis was applied to the gene sets reported by the studies selected for this review. Analysis was performed using the $\mathrm{R}$ package HTSanalyse $R$ [20]. Where reported, gene sets were extracted and combined according to the chemotherapy treatments applied to patients in each study. The two groups assessed were those studies where all patients were treated with platinum and taxane in combination, and those studies where

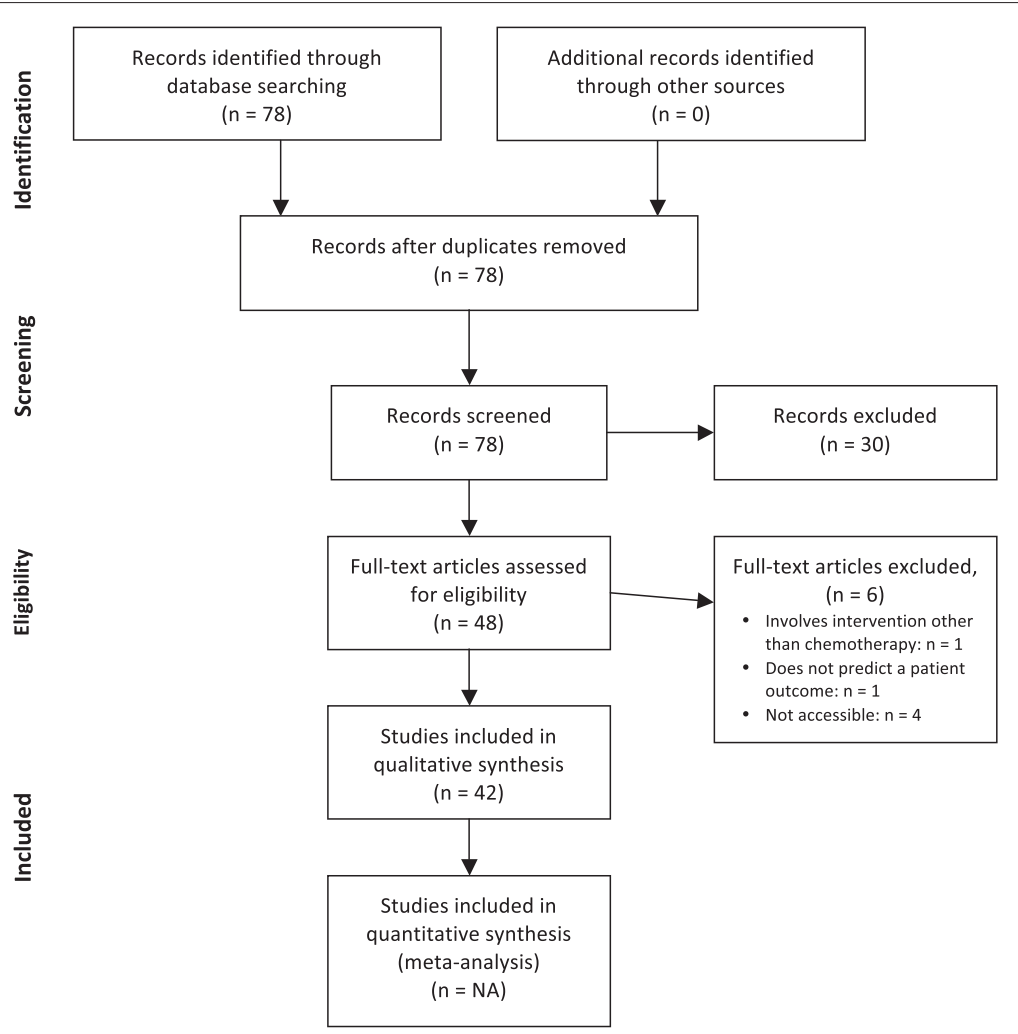

Figure 1 PRISMA search filtering flow diagram. The initial search results were filtered using titles and abstracts and, later, the full text to ensure the search criteria were fulfilled. Following filtering the number of papers included reduced from 78 to 42. 
Table 1 Journal and study information of papers included in the systematic review

\begin{tabular}{|c|c|c|c|c|}
\hline Study & Journal & No. samples & No. genes in study & No. genes in signature \\
\hline Jeong et al. [22] & Anticancer Res. & 487 & 612 & 388,612 \\
\hline Lisowska et al. [23] & Front. Oncol. & 127 & $>47000$ & 0 \\
\hline Roque et al. [24] & Clin. Exp. Metastasis & 48 & 1 & 1 \\
\hline Li et al. [3] & Oncol. Rep. & 44 & 1 & 1 \\
\hline Schwede et al. [25] & PLOS ONE & 663 & 2632 & 51 \\
\hline Verhaak et al. [26] & J. Clin. Invest. & 1368 & 11861 & 100 \\
\hline Obermayr et al. [27] & Gynecol. Oncol. & 255 & 29098 & 12 \\
\hline Han et al. [28] & PLOS ONE & 322 & 12042 & 349,18 \\
\hline Hsu et al. [29] & BMC Genomics & 168 & 12042 & 134 \\
\hline Lui et al. [30] & PLOS ONE & 737 & NS & 227 \\
\hline Kang et al. [31] & J. Nat. Cancer Inst. & 558 & 151 & 23 \\
\hline Gillet et al. [32] & Clin. Cancer Res. & 80 & 356 & 11 \\
\hline Ferriss et al. [33] & PLOS ONE & 341 & NS & 251,125 \\
\hline Brun et al. [34] & Oncol. Rep. & 69 & 6 & 0 \\
\hline Skirnisdottir and Seidal [35] & Oncol. Rep. & 105 & 3 & 2 \\
\hline Brenne et al. [36] & Hum. Pathol. & 140 & 1 & 1 \\
\hline Sabatier et al. [37] & Br. J. Cancer & 401 & NS & 7 \\
\hline Gillet et al. [38] & Mol. Pharmeceutics & 32 & 350 & $18,10,6$ \\
\hline Chao et al. [39] & BMC Med. Genomics & 6 & 8173 & NS \\
\hline Schlumbrecht et al. [40] & Mod. Pathol. & 83 & 7 & 2 \\
\hline Glaysher et al. [41] & Br. J. Cancer & 31 & 91 & $10,4,3,5,5,11,6,6$ \\
\hline Yan et al. [42] & Cancer Res. & 42 & 2 & 1 \\
\hline Yoshihara et al. [43] & PLOS ONE & 197 & 18176 & 88 \\
\hline Williams et al. [44] & Cancer Res. & 242 & NS & 15 to 95 \\
\hline Denkert et al. [45] & J. Pathol & 198 & NS & 300 \\
\hline Matsumura et al. [46] & Mol. Cancer Res. & 157 & 22215 & 250 \\
\hline Crijns et al. [47] & PLoS Medicine & 275 & 15909 & 86 \\
\hline Mendiola et al. [48] & PLOS ONE & 61 & 82 & 34 \\
\hline Gevaert et al. [49] & BMC Cancer & 69 & $\sim 24000$ & $\sim 3000$ \\
\hline Bachvarov et al. [50] & Int. J. Oncol. & 42 & 20174 & 155,43 \\
\hline Netinatsunthorn et al. [51] & BMC Cancer & 99 & 1 & 1 \\
\hline De Smet et al. [52] & Int. J. Gynecol. Cancer & 20 & 21372 & 3000 \\
\hline Helleman et al. [53] & Int. J. Cancer & 96 & NS & 9 \\
\hline Spentzos et al. [54] & J. Clin. Oncol. & 60 & NS & 93 \\
\hline Jazaeri et al. [55] & Clin. Cancer Res. & 40 & 40033,7585 & 85,178 \\
\hline Raspollini et al. [56] & Int. J. Gynecol. Cancer & 52 & 2 & 2 \\
\hline Hartmann et al. [57] & Clin. Cancer Res. & 79 & 30721 & 14 \\
\hline Spentzos et al. [58] & J. Clin. Oncol. & 68 & 12625 & 115 \\
\hline Selvanayagam et al. [59] & Cancer Genet. Cytogenet. & 8 & 10692 & NS \\
\hline Iba et al. [60] & Cancer Sci. & 118 & 4 & 1 \\
\hline Kamazawa et al. [61] & Gynecol. Oncol. & 27 & 3 & 1 \\
\hline Vogt et al. [62] & Acta Biochim. Pol. & 17 & 3 & 0 \\
\hline
\end{tabular}

If more than one value is given, the study used multiple different starting gene-sets or found multiple gene signatures. NS: Not Specified. 
Table 2 Tissue information of papers included in systematic review

\begin{tabular}{|c|c|c|}
\hline Study & Tissue source & $\%$ Cancerous tissue \\
\hline \multicolumn{3}{|l|}{ Jeong et al. [22] } \\
\hline Lisowska et al. [23] & Fresh-frozen & NS \\
\hline Roque et al. [24] & FFPE, Fresh-frozen & $\min .70 \%$ \\
\hline Li et al. [3] & FFPE & NS \\
\hline \multicolumn{3}{|l|}{ Schwede et al. [25] } \\
\hline \multicolumn{3}{|l|}{ Verhaak et al. [26] } \\
\hline Obermayr et al. [27] & Fresh-frozen, Blood & NS \\
\hline \multicolumn{3}{|l|}{ Han et al. [28] } \\
\hline \multicolumn{3}{|l|}{ Hsu et al. [29] } \\
\hline \multicolumn{3}{|l|}{ Lui et al. [30] } \\
\hline \multicolumn{3}{|l|}{ Kang et al. [31] } \\
\hline Gillet et al. [32] & Fresh-frozen & $\min .75 \%$ \\
\hline Ferriss et al. [33] & FFPE & $\min .70 \%$ \\
\hline Brun et al. [34] & FFPE & NS \\
\hline Skirnisdottir and Seidal [35] & FFPE & NS \\
\hline Brenne et al. [36] & Fresh-frozen effusion, Fresh-frozen & $\min .50 \%$ \\
\hline Sabatier et al. [37] & Fresh-frozen & $\min .60 \%$ \\
\hline Gillet et al. [38] & Fresh-frozen effusion & NS \\
\hline \multicolumn{3}{|l|}{ Chao et al. [39] } \\
\hline Schlumbrecht et al. [40] & Fresh-frozen & $\min .70 \%$ \\
\hline Glaysher et al. [41] & FFPE, Fresh & $\min .80 \%$ \\
\hline Yan et al. [42] & Fresh-frozen & NS \\
\hline Yoshihara et al. [43] & Fresh-frozen & $\min .80 \%$ \\
\hline \multicolumn{3}{|l|}{ Williams et al. [44] } \\
\hline Denkert et al. [45] & Fresh-frozen & NS \\
\hline Matsumura et al. [46] & Fresh-frozen & NS \\
\hline Crijns et al. [47] & Fresh-frozen & median $=70 \%$ \\
\hline Mendiola et al. [48] & FFPE & $\min .80 \%$ \\
\hline Gevaert et al. [49] & Fresh-frozen & NS \\
\hline Bachvarov et al. [50] & Fresh-frozen & $\min .70 \%$ \\
\hline Netinatsunthorn et al. [51] & FFPE & NS \\
\hline De Smet et al. [52] & Not specified & NS \\
\hline Helleman et al. [53] & Fresh-frozen & median =64\% \\
\hline Spentzos et al. [54] & Fresh-frozen & NS \\
\hline Jazaeri et al. [55] & FFPE, Fresh-frozen & NS \\
\hline Raspollini et al. [56] & FFPE & NS \\
\hline Hartmann et al. [57] & Fresh-frozen & $\min .70 \%$ \\
\hline Spentzos et al. [58] & Fresh-frozen & NS \\
\hline Selvanayagam et al. [59] & Fresh-frozen & $\min .70 \%$ \\
\hline Iba et al. [60] & FFPE, Fresh-frozen & NS \\
\hline Kamazawa et al. [61] & FFPE, Fresh-frozen & NS \\
\hline Vogt et al. [62] & None specified & NS \\
\hline
\end{tabular}

If more than one value is given, the study used tissue from multiple sources. NS: Not Specified. 
Table 3 Gene expression measurement techique information of papers included in systematic review

\begin{tabular}{|c|c|c|c|c|c|c|}
\hline Study & Immunohistochemistry & TaqMan array & q-RT-PCR & Commercial microarray & Custom microarray & RT-PCR \\
\hline Jeong et al. [22] & $x$ & $x$ & $x$ & $\checkmark$ & $x$ & $x$ \\
\hline Lisowska et al. [23] & $x$ & $x$ & $\checkmark$ & $\checkmark$ & $x$ & $x$ \\
\hline Roque et al. [24] & $\checkmark$ & $x$ & $\checkmark$ & $x$ & $x$ & $x$ \\
\hline Li et al. [3] & $\checkmark$ & $x$ & $x$ & $x$ & $x$ & $x$ \\
\hline Schwede et al. [25] & $x$ & $x$ & $x$ & $\checkmark$ & $x$ & $x$ \\
\hline Verhaak et al. [26] & $x$ & $x$ & $x$ & $\checkmark$ & $x$ & $x$ \\
\hline Obermayr et al. [27] & $x$ & $x$ & $\checkmark$ & $\checkmark$ & $x$ & $x$ \\
\hline Han et al. [28] & $x$ & $x$ & $x$ & $\checkmark$ & $x$ & $x$ \\
\hline Hsu et al. [29] & $x$ & $x$ & $x$ & $\checkmark$ & $x$ & $x$ \\
\hline Lui et al. [30] & $x$ & $x$ & $x$ & $\checkmark$ & $x$ & $x$ \\
\hline Kang et al. [31] & $x$ & $x$ & $x$ & $\checkmark$ & $x$ & $x$ \\
\hline Gillet et al. [32] & $x$ & $\checkmark$ & $x$ & $x$ & $x$ & $x$ \\
\hline Ferriss et al. [33] & $x$ & $x$ & $x$ & $x$ & $\checkmark$ & $x$ \\
\hline Brun et al. [34] & $\checkmark$ & $x$ & $x$ & $x$ & $x$ & $x$ \\
\hline Skirnisdottir and Seidal [35] & $\checkmark$ & $x$ & $x$ & $x$ & $x$ & $x$ \\
\hline Brenne et al. [36] & $x$ & $x$ & $\checkmark$ & $x$ & $x$ & $x$ \\
\hline Sabatier et al. [37] & $x$ & $x$ & $x$ & $\checkmark$ & $x$ & $x$ \\
\hline Gillet et al. [38] & $x$ & $\checkmark$ & $x$ & $x$ & $x$ & $x$ \\
\hline Chao et al. [39] & $x$ & $x$ & $x$ & $\checkmark$ & $x$ & $x$ \\
\hline Schlumbrecht et al. [40] & $\checkmark$ & $x$ & $\checkmark$ & $x$ & $x$ & $x$ \\
\hline Glaysher et al. [41] & $x$ & $\checkmark$ & $x$ & $x$ & $x$ & $x$ \\
\hline Yan et al. [42] & $\checkmark$ & $x$ & $x$ & $x$ & $x$ & $x$ \\
\hline Yoshihara et al. [43] & $x$ & $x$ & $\checkmark$ & $\checkmark$ & $x$ & $x$ \\
\hline Williams et al. [44] & $x$ & $x$ & $x$ & $\checkmark$ & $x$ & $x$ \\
\hline Denkert et al. [45] & $x$ & $x$ & $x$ & $\checkmark$ & $x$ & $x$ \\
\hline Matsumura et al. [46] & $\checkmark$ & $x$ & $\checkmark$ & $\checkmark$ & $x$ & $x$ \\
\hline Crijns et al. [47] & $x$ & $x$ & $\checkmark$ & $x$ & $\checkmark$ & $x$ \\
\hline Mendiola et al. [48] & $x$ & $\checkmark$ & $x$ & $x$ & $x$ & $x$ \\
\hline Gevaert et al. [49] & $x$ & $x$ & $x$ & $\checkmark$ & $x$ & $x$ \\
\hline Bachvarov et al. [50] & $x$ & $x$ & $\checkmark$ & $\checkmark$ & $x$ & $x$ \\
\hline Netinatsunthorn et al. [51] & $\checkmark$ & $x$ & $x$ & $x$ & $x$ & $x$ \\
\hline De Smet et al. [52] & $x$ & $x$ & $x$ & $x$ & $\checkmark$ & $x$ \\
\hline Helleman et al. [53] & $x$ & $x$ & $\checkmark$ & $x$ & $\checkmark$ & $x$ \\
\hline Spentzos et al. [54] & $x$ & $x$ & $x$ & $\checkmark$ & $x$ & $x$ \\
\hline Jazaeri et al. [55] & $\checkmark$ & $x$ & $x$ & $x$ & $\checkmark$ & $x$ \\
\hline Raspollini et al. [56] & $\checkmark$ & $x$ & $x$ & $x$ & $x$ & $x$ \\
\hline Hartmann et al. [57] & $x$ & $x$ & $x$ & $x$ & $\checkmark$ & $x$ \\
\hline Spentzos et al. [58] & $x$ & $x$ & $x$ & $\checkmark$ & $x$ & $x$ \\
\hline Selvanayagam et al. [59] & $x$ & $x$ & $x$ & $x$ & $\checkmark$ & $x$ \\
\hline lba et al. [60] & $\checkmark$ & $x$ & $\checkmark$ & $x$ & $x$ & $x$ \\
\hline Kamazawa et al. [61] & $x$ & $x$ & $\checkmark$ & $x$ & $x$ & $x$ \\
\hline Vogt et al. [62] & $x$ & $x$ & $x$ & $x$ & $x$ & $\checkmark$ \\
\hline
\end{tabular}


patients were given treatments other than platinum and taxane. The second group includes those given platinum as a single agent. Any studies reporting treatments from both groups were excluded, as were studies that did not report the chemotherapy treatments used. Kyoto Encyclopedia of Genes and Genomes (KEGG) terms were identified for each gene and gene set collection analysis was carried out, which applies hypergeometric tests and gene set enrichment analysis. A p-value cut-off of 0.0001 was used. Enrichment maps were then plotted, using the 30 most significant KEGG terms. P-values were adjusted using the 'BH' correction [21].

\section{Ethics statement}

Ethical approval was not required for this systematic review, which deals exclusively with previously published data.

\section{Results}

Tables 1, 2, 3, 4, 5 and 6 detail some key information regarding the studies included in the review. Table 1 contains the number of samples analysed, the number of genes considered for the model, and the resulting genes retained as the predictive gene signature. Table 2 provides information about the tissue used for gene expression measurements and whether the studies assessed the percent neoplastic tissue before measurement, and Table 3 details the gene expression measurement techniques used. Table 4 contains the reported histological types and stages of the samples processed by each study. Table 5 provides information on chemotherapy treatments undergone by patients, whether the model was prognostic or predictive, and whether the model was validated using either an independent set of samples or cross validation. Table 6 lists the outcome to be predicted, the modelling techniques applied, and the predictive ability of the resulting model.

\section{Tissue source}

For studies involving RNA extraction the tissue source is an important consideration, as RNA degradation and fragmentation could affect the results of techniques involving amplification. This is a notable issue in formalin fixed paraffin embedded (FFPE) tissue, due to the cross-linking of genetic material and proteins [63]. Of the 42 papers included in this review, the majority used fresh-frozen biopsy tissue. The numbers of each tissue source may be found in Table 7, and the tissue source used by individual papers may be found in Table 2. Nine papers did not use an RNA source directly as secondary data was used. Data sources were mostly other studies or data repositories, such as the TCGA dataset. Two studies did not specify the source tissue though extraction and expression measurement methods were detailed.
The majority of papers in this review used fresh-frozen tissue. This choice was likely made to minimise RNA degradation and hence improve measurement accuracy. Due to the risk of RNA degradation because of long storage times and the fixing process applied to FFPE tissue, it is often expected that FFPE tissue will be irreversibly cross-linked and fragmented. However, following investigation into RNA integrity when extracted from paired FFPE and fresh-frozen tissue, Rentoft et al. [64] found that for most samples up- and down-regulation of four genes was found to be the same whether measured in FFPE or fresh-frozen tissue. They concluded that, if samples were screened to ensure RNA quality, FFPE material can successfully provide RNA for gene expression measurement.

The use of fresh-frozen tissue in a research setting is not unusual, as can be seen from the fact that this tissue type was most popular in this review. However, for translational research expected to lead to a clinical test, this is not as reasonable. FFPE tissue is much more readily available, due to simpler acquisition and storage, and tissue is already taken for histological analysis. Therefore a model capable of using data obtained from FFPE tissue is much more likely to be applicable in a clinical setting.

Another important consideration is the proportion of neoplastic cells in the sample. For each paper the reported proportion may be seen in Table 2. Of the 42 papers, 14 reported that the proportion of cancerous cells was measured. This was usually done using hematoxylin and eosin stained histologic slides. It is important for the gene expression measurement that the tissue used contains a high proportion of neoplastic cells, and hence it is important that this pre-analytical variable is controlled. Of the studies in this review, those reporting the percentage cancerous cells were evenly distributed between FFPE and fresh-frozen tissues.

\section{Gene or protein expression quantification}

Of the studies highlighted by this review, there were four main techniques applied for gene or protein expression measurement: Probe-target hybridization microarrays, quantitative PCR, reverse transcription end-point-PCR, and immunohistochemical staining. Of these methods only immunohistochemistry measures protein expression, via classification of the level of staining, and the other methods quantify gene expression via measurement of mRNA copy number.

Methods involving probe-target hybridization are available commercially, and 19 of the 42 studies utilised these. For example the Affymetrix Human U133A 2.0 GeneChip and the Agilent Whole Human Genome Oligo Microarray were both used by multiple studies. Additionally, 7 studies used custom-made probe-target hybridization arrays. Probe-target hybridisation arrays generally measure thousands of genes and hence can provide a 
Table 4 Histology information of papers included in systematic review

\begin{tabular}{|c|c|c|}
\hline Study & Sub-type & Stage \\
\hline Jeong et al. [22] & Serous, Endometrioid, Adenocarcinoma & I, II, III, IV \\
\hline Lisowska et al. [23] & Serous, Endometrioid, Clear cell, Undifferentiated & II, III, IV \\
\hline Roque et al. [24] & Serous, Endometrioid, Clear cell, Undifferentiated, Mixed & IIIC, IV \\
\hline Li et al. [3] & Serous, Endometrioid, Clear cell, Mucinous, Transitional & II, III, IV \\
\hline Schwede et al. [25] & Serous, Endometrioid, Clear cell, Mucinous, Adenocarcinoma, OSE & I, II, III, IV \\
\hline Verhaak et al. [26] & NS & II, III, IV \\
\hline Obermayr et al. [27] & Serous, Non-serous & II, III, IV \\
\hline Han et al. [28] & Serous, Endometrioid, Clear cell, Mucinous, Mixed, Poorly differentiated & II, III, IV \\
\hline Hsu et al. [29] & NS & III, IV \\
\hline Lui et al. [30] & Serous & II, III, IV \\
\hline Kang et al. [31] & Serous & I, II, III, IV \\
\hline Gillet et al. [32] & Serous & III, IV \\
\hline Ferriss et al. [33] & Serous, Clear cell, Other & III, IV \\
\hline Brun et al. [34] & Serous, Endometrioid, Clear cell, Mucinous, Other & III, IV \\
\hline Skirnisdottir and Seidal [35] & Serous, Endometrioid, Clear cell, Mucinous, Anaplastic & I, II \\
\hline Brenne et al. [36] & Serous, Endometrioid, Clear cell, Undifferentiated, Mixed & II, III, IV \\
\hline Sabatier et al. [37] & Serous, Endometrioid, Clear cell, Mucinous, Undifferentiated, Mixed & I, II, III, IV \\
\hline Gillet et al. [38] & Serous & III, IV, NS \\
\hline Chao et al. [39] & NS & NS \\
\hline Schlumbrecht et al. [40] & Serous & III, IV \\
\hline Glaysher et al. [41] & Serous, Endometrioid, Clear cell, Mucinous, Mixed, Poorly differentiated & $I I I C, I V$ \\
\hline Yan et al. [42] & Serous, Endometrioid, Clear cell, Mucinous, Transitional & II, III, IV \\
\hline Yoshihara et al. [43] & Serous & III, IV \\
\hline Williams et al. [44] & Serous, Endometrioid, Undifferentiated & III, IV \\
\hline Denkert et al. [45] & Serous, Non-serous, Undifferentiated & I, II, III, IV \\
\hline Matsumura et al. [46] & Serous & I, II, III, IV \\
\hline Crijns et al. [47] & Serous & III, IV \\
\hline Mendiola et al. [48] & Serous, Non-serous & III, IV \\
\hline Gevaert et al. [49] & Serous, Endometrioid, Mucinous, Mixed & I, III, IV \\
\hline Bachvarov et al. [50] & Serous, Endometrioid, Clear cell & II, III, IV \\
\hline Netinatsunthorn et al. [51] & Serous & III, IV \\
\hline De Smet et al. [52] & Serous, Endometrioid, Mucinous, Mixed & I, III, IV \\
\hline Helleman et al. [53] & Serous, Endometrioid, Clear cell, Mucinous, Mixed, Poorly differentiated & I/II, III/IV \\
\hline Spentzos et al. [54] & Serous, Endometrioid, Clear cell, Mixed & I, II, III, IV \\
\hline Jazaeri et al. [55] & Serous, Endometrioid, Clear cell, Mixed, Undifferentiated, Carcinoma & II, III, IV \\
\hline Raspollini et al. [56] & Serous & IIIC \\
\hline Hartmann et al. [57] & Serous, Endometrioid, Mixed & II, III, IV \\
\hline Spentzos et al. [58] & Serous, Endometrioid, Clear cell, Mixed & I, II, III, IV \\
\hline Selvanayagam et al. [59] & Serous, Endometrioid, Clear cell, Undifferentiated & III, IV \\
\hline Iba et al. [60] & Serous, Endometrioid, Clear cell, Mixed & I, II, III, IV \\
\hline Kamazawa et al. [61] & Serous, Endometrioid, Clear cell & III, IV \\
\hline Vogt et al. [62] & NS & NS \\
\hline
\end{tabular}

Entries in bold indicate that the study data set was comprised of at least 80\% this type. NS: Not Specified. 
Table 5 Basic modelling and patient information of papers included in systematic review

\begin{tabular}{|c|c|c|c|c|}
\hline Study & $\begin{array}{l}\text { Patient prior chemotherapy } \\
\text { treatment }\end{array}$ & $\begin{array}{l}\text { Model accounts for the different } \\
\text { chemotherapies? }\end{array}$ & Prognostic or predictive? & Model validated? \\
\hline Jeong et al. [22] & Platinum-based & $\checkmark$ & Predictive & $\checkmark$ \\
\hline Lisowska et al. [23] & $\begin{array}{l}\text { Platinum/Cyclophosphamide, } \\
\text { Platinum/Taxane }\end{array}$ & $x$ & Prognostic & $\checkmark$ \\
\hline Roque et al. [24] & NS & $x$ & Prognostic & $x$ \\
\hline Li et al. [3] & $\begin{array}{l}\text { Platinum/Cyclophosphamide, } \\
\text { Platinum/Taxane }\end{array}$ & $x$ & Prognostic & $x$ \\
\hline Schwede et al. [25] & NS & $x$ & Prognostic & $\checkmark$ \\
\hline Verhaak et al. [26] & NS & $x$ & Prognostic & $\checkmark$ \\
\hline Obermayr et al. [27] & Platinum-based & $x$ & Prognostic & $x$ \\
\hline Han et al. [28] & Platinum/Paclitaxel & & Prognostic & $\checkmark$ \\
\hline \multirow[t]{2}{*}{ Hsu et al. [29] } & Platinum/Paclitaxel & & & \\
\hline & + additional treatments & $\checkmark$ & Prognostic & $\checkmark$ \\
\hline Lui et al. [30] & NS & $x$ & Prognostic & $\checkmark$ \\
\hline Kang et al. [31] & Platinum/Taxane & & Prognostic & $\checkmark$ \\
\hline Gillet et al. [32] & Carboplatin/Paclitaxel & & Prognostic & $\checkmark$ \\
\hline Ferriss et al. [33] & Platinum-based & $\checkmark$ & Predictive & $\checkmark$ \\
\hline Brun et al. [34] & NS & $x$ & Prognostic & $x$ \\
\hline Skirnisdottir and Seidal [35] & Carboplatin/Paclitaxel & & Prognostic & $x$ \\
\hline Brenne et al. [36] & NS & $x$ & Prognostic & $x$ \\
\hline Sabatier et al. [37] & Platinum-based & $x$ & Prognostic & $\checkmark$ \\
\hline Gillet et al. [38] & NS & $x$ & Prognostic & $\checkmark$ \\
\hline Chao et al. [39] & NS & $x$ & Prognostic & $x$ \\
\hline Schlumbrecht et al. [40] & Platinum/Taxane & & Prognostic & $x$ \\
\hline Glaysher et al. [41] & Platinum, Platinum/Paclitaxel & $\checkmark$ & Predictive & $\checkmark$ \\
\hline Yan et al. [42] & Platinum-based & $x$ & Prognostic & $x$ \\
\hline Yoshihara et al. [43] & Platinum/Taxane & & Prognostic & $\checkmark$ \\
\hline Williams et al. [44] & NS & $\checkmark$ & Predictive & $\checkmark$ \\
\hline Denkert et al. [45] & Carboplatin/Paclitaxel & & Prognostic & $\checkmark$ \\
\hline Matsumura et al. [46] & Platinum-based & $\checkmark$ & Predictive & $\checkmark$ \\
\hline \multirow[t]{2}{*}{ Crijns et al. [47] } & Platinum, Platinum/ & & & \\
\hline & $\begin{array}{l}\text { Cyclophosphamide, } \\
\text { Platinum/Paclitaxel }\end{array}$ & $\checkmark$ & Prognostic & $\checkmark$ \\
\hline Mendiola et al. [48] & Platinum/Taxane & & Prognostic & $\checkmark$ \\
\hline Gevaert et al. [49] & NS & $x$ & Prognostic & $\checkmark$ \\
\hline \multirow[t]{2}{*}{ Bachvarov et al. [50] } & Carboplatin/Paclitaxel, & & & \\
\hline & $\begin{array}{l}\text { Carboplatin/Cyclophosphamide, } \\
\text { Cisplatin/Paclitaxel }\end{array}$ & $x$ & Prognostic & $\checkmark$ \\
\hline Netinatsunthorn et al. [51] & Platinum/Cyclophosphamide & & Prognostic & $x$ \\
\hline De Smet et al. [52] & $\begin{array}{l}\text { Platinum/Cyclophosphamide, } \\
\text { Platinum/Paclitaxel }\end{array}$ & $x$ & Prognostic & $\checkmark$ \\
\hline Helleman et al. [53] & $\begin{array}{l}\text { Platinum/Cyclophosphamide, } \\
\text { Platinum-based }\end{array}$ & $x$ & Prognostic & $\checkmark$ \\
\hline Spentzos et al. [54] & Platinum/Taxane & & Prognostic & $\checkmark$ \\
\hline
\end{tabular}


Table 5 Basic modelling and patient information of papers included in systematic review (Continued)

\begin{tabular}{|c|c|c|c|c|}
\hline Jazaeri et al. [55] & $\begin{array}{l}\text { Carboplatin/Paclitaxel, Cis- } \\
\text { platin/Cyclophosphamide, } \\
\text { Carboplatin/Docetaxel, } \\
\text { Carboplatin }\end{array}$ & $x$ & Prognostic & $\checkmark$ \\
\hline Raspollini et al. [56] & $\begin{array}{l}\text { Cisplatin/Cyclophosphamide, } \\
\text { Carbo- } \\
\text { platin/Cyclophosphamide, } \\
\text { Carboplatin/Paclitaxel }\end{array}$ & $x$ & Prognostic & $x$ \\
\hline Hartmann et al. [57] & $\begin{array}{l}\text { Cisplatin/Paclitaxel, Carbo- } \\
\text { platin/Paclitaxel }\end{array}$ & $x$ & Prognostic & $\checkmark$ \\
\hline Spentzos et al. [58] & Platinum/Taxane & & Prognostic & $\checkmark$ \\
\hline Selvanayagam et al. [59] & $\begin{array}{l}\text { Cisplatin/Cyclophosphamide, } \\
\text { Carbo- } \\
\text { platin/Cyclophosphamide, } \\
\text { Cisplatin/Paclitaxel }\end{array}$ & $x$ & Prognostic & $\checkmark$ \\
\hline Iba et al. [60] & Carboplatin/Paclitaxel & & Prognostic & $x$ \\
\hline Kamazawa et al. [61] & Carboplatin/Paclitaxel & & Prognostic & $x$ \\
\hline Vogt et al. [62] & $\begin{array}{l}\text { Etoposide, } \\
\text { Paclitaxel/Epirubicin, } \\
\text { Carboplatin/Paclitaxel }\end{array}$ & $\checkmark$ & Predictive & $x$ \\
\hline
\end{tabular}

If more than one value is given, the study included patients treated with different treatments. NS: Not Specified.

wealth data per sample. TaqMan microfluidic arrays or quantitative-PCR were used by 16 studies. These techniques are typically used for smaller panels of genes. The TaqMan arrays for example may contain up to 384 genes per array. These methods are more targeted and hence the price per sample is usually lower.

Immunohistochemistry is a more labour-intensive technique, requiring staining for each gene considered, and hence was mostly only used by studies using small numbers of genes. This technique, which is semi-quantitative due to the scoring systems employed, also suffers from a lack of standardisation of procedures. Of the 11 papers using this technique, the maximum number of genes analysed was seven, and the mean number of genes assessed was 2.8. Although these studies provide useful information regarding the correlation of particular genes with outcome, the small numbers of genes is likely to result in an incomplete gene signature and low predictive power.

Several of the papers utilising quantifiable techniques used an alternative method or replicates to obtain a measure of the assay variability. Five papers involving commercial or custom microarrays also used reverse transcription PCR (RT-PCR) to measure the expression of a small number of genes for comparison and one study used samples run in duplicate to calculate the coefficient of variation. Of the studies using TaqMan microfluidic arrays, two used samples run in duplicate to obtain the coefficient of variation. However, even fewer papers reported a metric representing the level of variability found. Two studies reported a coefficient of variation; Glaysher et al. [41] reported $\mathrm{CoV}=2 \%=0.02$ for TaqMan arrays and Hartmann et al. [57] reported $\mathrm{CoV}=$
0.2 for their custom microarray. Another two reported Spearman's or Pearson's $r$ coefficients of correlation between microarray and RT-PCR results. Yoshihara et al. [43] gave Pearson $r$ values ranging from 0.5 to 0.8 , and Crijns et al. [47] gave Spearman's $r$ values between -0.6 and -0.9 .

\section{Histology}

Table 4 details the histology (types and stages) of the patient samples used by each study. As may be seen, the majority of studies were heterogeneous with respect to the types of cancer included. However, 23 of the 42 studies used at least $80 \%$ serous samples, suggesting that the majority of information contributed to the gene signatures of these studies is related to the mechanisms and pathways in serous cancer. In the authors' opinion it is important to identify the histologies of patient samples: although treatment is currently the same across types, response to chemotherapy has been found to vary $[9,65,66]$. It therefore may be advisable for future studies to include histological information when developing models predicting chemotherapy response.

\section{Chemotherapy}

Table 5 lists the chemotherapy treatments undergone by patients in each study. The 10 papers labelled NS did not specify the regimen applied, though the patients did have chemotherapy. These cohorts cannot therefore be assumed to be homogeneous with respect to patient chemotherapy treatment. All studies that specified the chemotherapy regimen undergone by patients noted at least one platinum-based treatment. Of these, 24 included 
Table 6 Basic modelling information of papers included in systematic review

\begin{tabular}{lll}
\hline Study & Prediction & Prediction method \\
\hline Jeong et al. [22] & Overall Survival & Student's T test, Hierarchical clus- \\
& tering, Compound covariate pre- \\
& dictor algorithm, Cox proportional \\
& hazards regression, Kaplan-Meier \\
& curves, Log-rank test, ROC analysis
\end{tabular}

Lisowska et al. [23]

Roque et al. [24]

Li et al. [3]

Schwede et al. [25]

Verhaak et al. [26]

Obermayr et al. [27]

Han et al. [28]

Hsu et al. [29]

Lui et al. [30]

Kang et al. [31]

Gillet et al. [32]

Ferriss et al. [33]
Chemoresponse, Disease-Free Survival, Overall Survival

Overall Survival

Chemoresponse (chemoresistant vs. chemosensitive)

Stem cell-like subtype, Disease-Free Survival, Overall Survival

Poor Prognosis vs. Good Prognosis

Disease-Free Survival, Overall Survival

Complete Response or Progressive Disease

Progression-Dree Survival

Chemosensitivity, Overall Survival, Progression-Dree Survival

Overall Survival, Progression-Free Survival, Recurrence-Free Survival

Overall Survival, Progression-Free Survival

Overall Survival
Support vector machines, KaplanMeier curves, Log-rank test

Kaplan-Meier curves, Log-rank test, Student's T test

Correlation of p-CFL1 staining and chemoresponse

ISIS unsupervised bipartitioning, Diagonal linear discriminant analysis, Gaussian mixture modelling, Kaplan-Meier curves, Log-rank test

Significance analysis of microarrays, Single sample gene set enrichment analysis, Kaplan-Meier curves, Logrank test

Kaplan-Meier curves, Cox proportional hazards regression, $\chi^{2}$ test

Supervised principal component method

Semi-supervised hierarchical clustering

Predictive score using weighted voting algorithm, Kaplan-Meier curves, Log-rank Test, Cox proportional hazards regression

Kaplan-Meier curves, Log-rank test, Cox proportional hazards regression, Pearson correlation coefficient

Supervised principle components method, Cox proportional hazards regression, Kaplan-Meier curves, Log-rank test

COXEN coefficient, Mann-Whitney $U$ test, ROC analysis, Unsupervised Hierarchical Clustering

\section{Predictive ability}

'Taxane-based treatment significantly affected OS for patients in the YA subgroup (3 year rate: 74.4\% with taxane vs. 37.9\% without taxane, $\mathrm{p}=0.005$ by log-rank test)', 'estimated hazard ratio for death after taxane-based treatment in the YA subgroup was 0.5 (95\% $C l=0.31--0.82, p=0.005)$

No genes found to be significant in the training set were significant in the test set, for chemoresponse, DFS or OS

'OS was predicted by increased class III $\beta$-tubulin staining by both tumor (HR3.66, 96\% Cl=1.11-12.1, $p=0.03)$ and stroma (HR4.53, 95\% $C l=1.28-16.1, p=0.02)^{\prime}$

'immunostaining of p-CFL1 was positive in $77.3 \%$ of chemosensitive and in $95.9 \%$ of the chemoresistant ${ }^{\prime}$ ( $p=0.014, U=157.5)$

OS ( $p$ values): Dressman $=0.0354$ Crijns $=0.021$, Tothill $=4.4 E-7$

Good or Poor prognosis, likelihood ratio $=44.63$

'The presence of CTCs six months after completion of the adjuvant chemotherapy indicated relapse within the following six months with $41 \%$ sensitivity, and relapse within the entire observation period with $22 \%$ sensitivity (85\% specificity)

349 gene signature: $\mathrm{ROC}$ AUC= 0.702, $p=0.022$. 18 gene: $\mathrm{ROC}$ $\mathrm{AUC}=0.614, p=0.197$.

Good Response vs. Poor Response, $p=0.021$

Response of 26 of 35 patients in an independent data set was correctly predicted, patients in the lowscoring group exhibited poorer PFS $(H R=0.43, p=0.04)$, ROC AUC = 0.90(0.86-0.95)

Berchuck dataset: $H R=0.33,95 \%$ $C l=0.13-0.86, p=0.013$; Tothill dataset: $H R=0.61,95 \% \mathrm{Cl}=$ $0.36-0.99, p=0.044$

'An 11-gene signature whose measured expression significantly improves the power of the covariates to predict poor survival' $(p<0.003)$

Carboplatin: sensitivity $=0.906$, specificity $=0.174$, PPV $=60 \%$, NPV $=57 \%$ (UVA-55 validation set) 
Table 6 Basic modelling information of papers included in systematic review (Continued)

Brun et al. [34] 2-year Disease-Free Survival

Skirnisdottir and

Seidal [35]

Brenne et al. [36]

OC or MM, Progression-Free Survival, Overall Survival

Sabatier et al. [37]

Gillet et al. [38]

Schlumbrech et al. [40]

Glaysher et al. [41]

Yan et al. [42]

Yoshihara et al. [43]

Williams et al. [44]

Denkert et al. [45]

Matsumura et al. [46]
Progression-Free Survival, Overall Survival

Overall Survival, Progression-Free Survival, Treatment Response

Chemoresistance

Overall Survival, Recurrence-Free Survival

Chemosensitivity

Chemosensitivity

Progression-Free Survival

Overall Survival

Overall Survival

Taxane sensitivity, Overall Survival
Student's $T$ test, Principal component analysis, Concordance index, Kaplen-Meier curves, Log-rank test

$\chi^{2}$ test, Kaplan-Meier curves, Logrank test, Logistic regression, Cox proportional hazards regression

Mann-Whitney U test, Kaplan-Meier curves, Log-rank test, Cox proportional hazards regression

Cox proportional hazards regression, Pearson's coefficient correlation score

Linear regression, Hierarchical clustering, Kaplan-Meier curves, Logrank test

Interaction and expression networks for pathway identification, pathway intersections, betweenness and degree centrality, Student's T test

Linear regression, Logistic regression, Cox proportional hazards regression, Kaplan-Meier curves, Unsupervised cluster analysis, Logrank test, Mann-Whitney $U$ test, $\chi^{2}$ test

AIC gene selection, Multiple linear regression

ANOVA, Student's T test, MannWhitney $U$ test

Cox proportional hazards regression, Ridge regression, Prognostic index, ROC analysis, Kaplan-Meier curves, Log-rank test

COXEN score, Kaplan-Meier curves, Student's T test, ROC analysis, Spearman's rank correlation coefficient, Logistic regression, Log-rank test

Semi-supervised analysis via Cox scoring, Principal components analysis, Kaplan-Meier curves, Logrank test, Cox proportional hazards regression

Hierarchical clustering, KaplanMeier curves, Log-rank test
No genes were found to have prognostic value

p53-status $(O R=4.123, p=0.009$; $H R=2.447, p=0.019$ ) was a significant and independent factor for tumor recurrence and DFS.

Cox Multivariate Analysis: EHF mRNA expression in prechemotherapy effusions was an independent predictor of PFS $(p=0.033$, relative risk $=4.528)$

Favourable vs. Unfavourable: 'sensitivity $=61.6 \%$, specificity $=62.4 \%$, $O R=2.95 \% C l=1.7-4.2 ; p=$ $6.1 \times 10^{-06}$, Fisher's exact test'

' 6 gene signature alone can effectively predict the progression-free survival of women with ovarian serous carcinoma (log-rank $p=$ $0.002)^{\prime}$

No statistical measure available. Many genes identified have previously been found experimentally

'Greater EIG121 expression was associated with shorter time to recurrence $(H R=1.13$ $(C l=1.02-1.26), p=0.021)^{\prime}$, 'Increased expression of EIG121 demonstrated a statistically significant association with worse OS $(H R=1.21$ (C/1.09-1.35) $p<0.001)^{\prime}$

Cisplatin: $R_{\text {adj }}^{2}=0.836, p<0.001$

'Immunostaining scores [Annexin A3] are significantly higher in platinum-resistant tumors $(p=0.035)^{\prime}$

'Prognostic index was an independent prognostic factor for PFS time $(H R=1.64, p=0.0001)^{\prime}$, sensitivity $=64.4 \%$, specificity $=69.2 \%$

Carboplatin and Taxol: sensitivity = $77 \%$, specificity $=56 \%, P P V=71 \%$, $N P V=78 \%$

Duke et al.: 'clinical outcome is significantly different depending on the OPI ( $p=0.021)$, with an HR of $1.7(\mathrm{Cl} 1.1-2.6)^{\prime}$

'Patients in the YY1-High cluster who were treated with paclitaxel showed improved survival compared with the other groups ( $p=$ $0.010)^{\prime}$ 
Table 6 Basic modelling information of papers included in systematic review (Continued)

\begin{tabular}{|c|c|}
\hline Crijns et al. [47] & Overall Survival \\
\hline $\begin{array}{l}\text { Mendiola et al. } \\
\text { [48] }\end{array}$ & $\begin{array}{l}\text { Progression-Free Survival, Overall } \\
\text { Survival }\end{array}$ \\
\hline Gevaert et al. [49] & Platin Resistance/Sensitivity, Stage \\
\hline $\begin{array}{l}\text { Bachvarov et al. } \\
\text { [50] }\end{array}$ & Chemoresistance \\
\hline $\begin{array}{l}\text { Netinatsunthorn } \\
\text { et al. [51] }\end{array}$ & $\begin{array}{l}\text { Overall Survival, Recurrence-Free } \\
\text { Survival }\end{array}$ \\
\hline De Smet et al. [52] & $\begin{array}{l}\text { Stage I vs. Advanced stage, Platin- } \\
\text { sensistive vs. Platin-resistant }\end{array}$ \\
\hline $\begin{array}{l}\text { Helleman et al. } \\
\text { [53] }\end{array}$ & $\begin{array}{l}\text { Chemoresponse (responder vs. } \\
\text { non-responder) }\end{array}$ \\
\hline $\begin{array}{l}\text { Spentzos et al. } \\
\text { [54] }\end{array}$ & $\begin{array}{l}\text { Chemoresponse (pathological-CR } \\
\text { or PD), Disease-Free survival, Overall } \\
\text { Survival }\end{array}$ \\
\hline
\end{tabular}

Jazaeri et al. [55] Clinical response

Raspollini et al. [56]

Hartmann et al. [57]

Spentzos et al. [58]

Selvanayagam et al. [59]

Iba et al. [60]

Kamazawa et al. [61]

Vogt et al. [62]
Supervised principal components method, Cox proportional hazards regression, Kaplan-Meier curves, Log-rank test, $\chi^{2}$ test

Kaplan-Meier curves, Log-rank test, AIC-based model selection, ROC curves, Cox proportional hazards regression

Principal component analysis, Least squares support vector machines

Hierarchical Clustering, Support vector machines

Kaplan-Meier curves, Cox proportional hazards regression

Principal component analysis, Least squares support vector machines

Class prediction, Hierarchical clustering, Principal component analysis

Class prediction analysis, Compound covariate algorithm, Average linkage hierarchical clustering, Kaplan-Meier curves, Log-rank test, Cox proportional hazards regression

Class prediction

Univariate logistic regression, $\chi^{2}$ test

Support vector machine, KaplanMeier curves, Log-rank test, average linkage clustering

Supervised pattern recognition/ class prediction, Kaplan-Meier curves, Log-rank test, Cox proportional hazards regression

Supervised voice-pattern recognition algorithm (clustering)

Kaplan-Meier curves, Log-rank test, Cox propotionate hazards regression, ROC analysis, $\chi^{2}$ test, Student's T test, Mann-Whitney $U$ test

Defined threshold expressionto divide responders and non-responders

Correlation of AUC from in-vitro ATP-CVA and gene expression
OSP: (High-risk vs. low-risk) $H R=$ $1.940, C l=1.190-3.163, p=0.008$

OS: sensitivity $=87.2 \%$, specificity $=$ $86.4 \%$

Platin-Resistance/Sensitivity: sensitivity $=67 \%$, specificity $=40 \%$, accuracy $=51.11 \%$

No prediction metric applied

OS: $H R=1.98,95 \% \mathrm{Cl}=1.28-3.79$, $p=0.0138 ;$ RFS: $H R=3.36,95 \%$ $C l=1.60-7.03, p=0.0017$

Estimated Classification Accuracy: Stage I vs Advanced Stage $=100 \%$, Platin-sensitive vs. Platin-resistant $=$ $76.9 \%$

Test set: $P P V=24 \%, N P V=97 \%$ sensitivity $=89 \%$, specificity $=59 \%$

Cox PH (resistant vs. sensitive): Recurrence $H R=2.7(95 \% \mathrm{Cl}=$ 1.2-6.1), Death $H R=3.9(95 \% \mathrm{Cl}=$ 3.1-11.4)

9 most significantly differentially expressed genes, primary chemoresistant vs. primary chemosensitive: accuracy $=77.8 \%$

COX-2: $O R=0.23,95 \% \mathrm{Cl}=$ $0.06-0.77, p=0.017$; MDR1: $O R=$ $0.01,95 \% \mathrm{Cl}=0.002-0.09, p=<$ 0.0005

Accuracy $=86 \%, P P V=95 \%$, $N P V=67 \%$

Unfavourable vs. Favourable OS : $(\mathrm{CPH}) \mathrm{HR}=4.6,95 \% \mathrm{Cl}=2.0-10.7$, $p=0.0001$

$P P V=1, N P V=1$

'Patients with c-myc expression of over 200 showed a significantly better 5-year survival rate $(69.8 \%$ vs. $43.5 \%)^{\prime}, p<0.05$

MDR-1 (all samples): specificity = $95 \%$, sensitivity $=100 \%$, predictive value $=96 \%$

All $p$ values for correlation of drugs and genes were $>0.05$

If more than one value is given, the study used multiple different prediction methods or predicted more than one endpoint. 
Table 7 Numbers of studies using various mRNA sources

\begin{tabular}{cc}
\hline mRNA source & Number of studies \\
\hline FFPE tissue & 12 \\
Fresh-frozen tissue & 22 \\
Fresh-frozen effusion & 2 \\
Fresh tissue & 1 \\
Blood & 1 \\
Not used & 9 \\
Not specified & 2 \\
\hline
\end{tabular}

patients treated with a platinum-taxane combination and 10 with a cyclophosphamide-platinum combination. It is important to note that 19 of the 42 papers stated the population was heterogeneous with regards to chemotherapy treatments and, of those that did, only 8 included patient treatment history as a feature of the study. The aims of the majority of the studies were to identify genes of which the expression may be used to predict survival time, or prognosis. As already noted, the presence of resistance to the chemotherapy agent administered will dramatically affect the survival of a patient. It is therefore reasonable to expect the gene signatures identified to include genes responsible for chemoresistance, which will depend on the mechanism of action of the drug. Using a heterogeneous cohort in terms of chemotherapy treatment may then be causing problems with the identification of a minimal predictive gene set.

\section{End-point to be predicted}

As may be expected, there was variation between the end-point chosen by studies for prediction. Popular endpoints include overall survival, progression-free survival and response to chemotherapy. The endpoints considered by each study may be found in Table 6 . Of these some are clinical endpoints, such as overall survival, others use non-clinical endpoints, such as response to chemotherapy, many of which are considered to be surrogates for overall survival. For cancer studies, overall survival is considered to be the most reliable and is the variable that is of most interest when considering the effect of an intervention.

\section{Model development}

Within this review, many different modelling techniques were used to identify an explanatory gene signature to predict patient outcome. The most popular was Cox proportional hazards regression, which was applied by 17 studies. This was closely followed by hierarchical clustering, which was used by 11 studies. All other methods were used by 8 or fewer studies. In total 24 different types of modelling techniques were applied, ranging from statistical tests such as Student's T test and Mann-Whitney U test, to logistic regression, to ridge regression. Table 8 lists the modelling techniques identified and the number of studies that employed them. It is of interest that most of the techniques applied are forms of classification. These methods result in samples being assigned to groups, such as 'good prognosis' and 'poor prognosis'. Whilst this may be useful in some settings, for a clinically-applicable tool a regression technique may be more appropriate as it will provide a value, such as a likelihood of relapse, rather than simply a class. Techniques in Table 8 capable of a numeric prediction include logistic and linear regression, Cox proportional hazards regression, and ridge regression.

Jointly with the modelling methods identified above, 23 of the 42 studies implemented Kaplan-Meier curves to visualise the survival of the patient classes identified by the models. This enables the difference in survival between classes, for example 'good prognosis' and 'poor prognosis', to be seen and assessed. The application of a log-rank test assesses the separation of the curves and identifies whether there is a statistically significant difference in survival distribution

Table 8 Key modelling techniques applied by studies in the review

\begin{tabular}{|c|c|}
\hline Technique & Number of papers \\
\hline Cox proportional hazards regression & 17 \\
\hline Hierarchical clustering & 11 \\
\hline Principal components analysis & 8 \\
\hline Student's T test & 7 \\
\hline Scoring algorithm & 6 \\
\hline Support Vector Machines & 5 \\
\hline Correlation coefficients & 5 \\
\hline Mann-Whitney $U$ test & 5 \\
\hline$\chi^{2}$ test & 5 \\
\hline ROC analysis & 5 \\
\hline Class prediction & 4 \\
\hline Logistic regression & 3 \\
\hline Linear regression & 3 \\
\hline AIC gene selection & 2 \\
\hline Concordance index & 1 \\
\hline Pathway interaction networks & 1 \\
\hline ANOVA & 1 \\
\hline Expression threshold identified & 1 \\
\hline Gene set enrichment analysis & 1 \\
\hline Linear discriminant analysis & 1 \\
\hline ISIS bipartitoning & 1 \\
\hline Gaussian mixture modelling & 1 \\
\hline Significance analysis of microarrays & 1 \\
\hline Ridge regression & 1 \\
\hline
\end{tabular}


between the classes. It should be noted that, although this gives an idea of separation of classes achieved by the model, the model results must still be compared with known outcomes to check positive and negative predictive power. This step was missing in several papers, such as Gillet et al. [38], where the p value returned by the log-rank test is given as the measure of model success.

It is important to highlight the difference between prognostic and predictive models. A prognostic model is one capable of predicting prognosis, such as survival time, using patient information and biomarkers and does not vary between different treatment options. In contrast, a predictive model is one able to predict the effect of a treatment on patient prognosis $[67,68]$. It is therefore clear that, although prognostic models may be useful for research purposes and when one treatment option is available (such as the standard platinum-taxane combination), predictive models have a much greater part to play in stratified medicine where the aim is to identify the most appropriate treatment on a patient-by-patient basis. In order for a model to be predictive, the effects of multiple treatments must be considered and the response compared with the biomarker status. Classification of the studies as prognostic or predictive may be seen in Table 5 . Of the papers identified by this review, only a minority considered the effects of chemotherapy treatment on the predicted outcome and hence could be considered predictive. Glaysher et al. [41] and Vogt et al. [62] produced separate models for various treatments, allowing the effects of different drugs and combinations to be compared. Both studies applied drugs in vitro to cultured tissue to measure response to chemotherapy. This was combined with gene expression measurements to form the model training data set. In this way the same patient samples may be used to create a set of models predicting response to a variety of drugs. These models are therefore predictive rather than prognostic. Alternatively, models may be trained on sets of patients split by treatments undergone, which would lead to treatment-specific models predicting response to the particular drug. This method was used by Jeong et al. [22], Ferriss et al. [33], Williams et al. [44] and Matsumura et al. [46]. Additionally, the use of a model variable specifying patient treatment history could allow these models to be combined onto one using a single training set of all patients. The model may then be passed a variable specifying the drug of interest for resistance prediction. A simple version of this method was implemented by Crijns et al. [47], who included a feature for whether a patient was treated with paclitaxel. It is clear that the integration of patient chemotherapy treatment into these models is underused, and it is likely to be beneficial for this to be incorporated into future research.

\section{Genes identified}

Of the 42 papers in this review, 32 provided full or partial lists of the genes identified by their models. Of the remainder, it was common that the gene sets were large or that the genes were not explicitly identified by the model, as is the case with modelling techniques such as principal components analysis.

In total across the papers, 1298 unique genes were selected by models and of these $93.53 \%$ were found by only one paper. The most commonly chosen gene was selected by only four papers. Table 9 shows the numbers and percentages of genes chosen by one to four papers.

A list of the genes identified by the papers in the review may be found in Table 10 .

It is clear that the gene sets selected by the studies are very different and there is very little overlap. The genes chosen by two or more studies may be seen in Table 11 . Many of these genes are known to have links to cancer, which may suggest that these genes are therefore implicated in ovarian cancer. It is possible that, although the genes selected varied, they in fact represent similar mechanisms. This could occur if there are large sets of highly covariate genes representing particular cellular processes and the genes in the signatures were simply random selections from these gene sets. The same gene being selected by multiple papers would then be unlikely, although the same information contribution would be made. It may then be more informative to assess and compare the mechanisms controlled by the genes chosen as part of the models.

\section{Gene set enrichment}

The gene sets reported by the studies identified in this review were assessed to identify whether certain biological pathways and mechanisms featured more prominently according to the genes selected. Studies were split by chemotherapy treatments recieved by the patients, and the groups identified were platinum and taxane, and other treatments (such as platinum, cyclophosphamide and combinations). Studies that did not specify the chemotherapy treatments used were excluded. Studies falling into the platinum and taxane group were Han et al. [28], Kang et al. [31], Gillet et al. [32], Skirnisdottir and

Table 9 Numbers and percentages of genes featured in the gene sets of various numbers of papers

\begin{tabular}{lll}
\hline $\begin{array}{l}\text { Number of papers } \\
\text { identifying a gene }\end{array}$ & Number of genes & Percent of genes \\
\hline 1 & 1214 & $93.53 \%$ \\
2 & 78 & $6.01 \%$ \\
3 & 5 & $0.385 \%$ \\
4 & 1 & $0.08 \%$ \\
\hline
\end{tabular}


Table 10 List of genes reported by studies included in this review

\begin{tabular}{|c|c|c|c|c|c|}
\hline $\mathrm{A} 1 \mathrm{BG}$ & CHPF2 & FSCN1 & LRRC16B & PKD1 & SOBP \\
\hline A2M & CHRDL1 & FXYD6 & LRRC17 & PKHD1 & SORBS3 \\
\hline AADAC & CHRNE & FZD4 & LRRC59 & PLA2G7 & SOS1 \\
\hline AAK1 & CHST6 & FZD5 & LRSAM1 & PLAA & SOX12 \\
\hline ABCA13 & CHTOP & G0S2 & LSAMP & PLAU & SOX21 \\
\hline $\mathrm{ABCA} 4$ & CIAPIN1 & G3BP1 & LSM14A & PLAUR & SPANXD \\
\hline ABCB1 & $\mathrm{ClB} 1$ & GABRP & LSM3 & PLCB3 & SPATA13 \\
\hline ABCB10 & $\mathrm{CIB} 2$ & GAD1 & LSM7 & PLEC & SPATA18 \\
\hline ABCB11 & CIITA & GALNT10 & LSM8 & PLEK & SPATA4 \\
\hline ABCB7 & CILP & GAP43 & LTA4H & PLIN2 & SPC25 \\
\hline $\mathrm{ABCC} 3$ & CITED2 & GART & LTB & PLS1 & SPDEF \\
\hline ABCC5 & CKLF & GATAD2A & LTK & PMM1 & SPEN \\
\hline ABCD2 & CLCA1 & $\mathrm{GCH} 1$ & LUC7L2 & PMP22 & SPHK2 \\
\hline ABCG2 & CLCNKB & GCHFR & LY6K & PMVK & SPOCK2 \\
\hline ABLIM1 & CLDN10 & GCM1 & LY96 & PNLDC1 & SPTBN2 \\
\hline ACADVL & CLIP1 & GDF6 & LZTFL1 & PNLIPRP2 & SRC \\
\hline ACAT2 & CNDP1 & GFRA1 & MAB21L2 & PNMA5 & SREBF2 \\
\hline ACKR2 & CNKSR3 & GGCT & MAD2L2 & POFUT2 & SRF \\
\hline ACKR3 & CNN2 & GGT1 & MAGEE2 & POLH & SRRM1 \\
\hline $\mathrm{ACO} 2$ & CNOT8 & GJB1 & MAGEF1 & POLR3K & SRSF3 \\
\hline ACOT13 & CNTFR & GLRX & MAK & POMP & SSR1 \\
\hline $\mathrm{ACP} 1$ & cofilin1 & GMFB & MAMLD1 & POU2AF1 & SSR2 \\
\hline ACRV1 & COL10A1 & GMPR & MANF & POU5F1 & SSUH2 \\
\hline ACSM1 & COL21A1 & GNA11 & MAP6D1 & PPAP2B & SSX2IP \\
\hline ACSS3 & COL3A1 & GNAO1 & MAPK1 & PPAT & ST6GALNAC1 \\
\hline ACTA2 & COL4A4 & GNAZ & MAPK1IP1L & PPCDC & STC2 \\
\hline ACTB & COL4A6 & GNG4 & MAPK3 & PPCS & STK38 \\
\hline ACTBL3 & COL6A1 & GNG7 & MAPK8IP3 & PPFIA3 & STX12 \\
\hline ACTG2 & COL7A1 & GNL2 & MAPK9 & PPIC & STX1B \\
\hline ACTR3B & COX8A & GNMT & MAPKAP1 & PPIE & STX7 \\
\hline ACTR6 & CPD & GNPDA1 & MAPKAPK2 & PPP1R1A & STXBP2 \\
\hline ADAMDEC 1 & CPE & GOLPH3 & MARCKS & PPP1R1B & STXBP6 \\
\hline ADAMTS5 & CPEB1 & GPIHBP1 & MARK4 & PPP1R2 & SUB1 \\
\hline ADIPOR2 & CRCT1 & GPM6B & MATK & PPP1R26 & SULT1C2 \\
\hline ADK & CREB5 & GPR137 & $\mathrm{MB}$ & PPP2R3C & SULT2B1 \\
\hline AEBP1 & CRYAB & GPT2 & MBOAT7 & PPP2R5C & SUPT5H \\
\hline AF050199 & CRYBB1 & GPX2 & MCF2L & PPP2R5D & SUSD4 \\
\hline AF052172 & CRYL1 & GPX3 & MCL1 & PPP4R4 & SUV420H1 \\
\hline AFM & CRYM & GPX8 & MCM3 & PPP6R1 & $\mathrm{SV} 2 \mathrm{C}$ \\
\hline AFTPH & CSE1L & GRAMD1B & $\mathrm{MDC} 1$ & PRAP1 & SYNM \\
\hline AGFG1 & CSPP1 & GRB2 & MDFI & PRELP & SYT1 \\
\hline AGR2 & CSRP1 & GRK6 & MDK & PRKAB1 & SYT11 \\
\hline AGT & CSRP3 & GRM2 & MDR-1 & PRKCH & SYT13 \\
\hline AIPL1 & CST6 & GRPEL1 & MEA1 & PRKCl & TAC3 \\
\hline
\end{tabular}


Table 10 List of genes reported by studies included in this review (Continued)

\begin{tabular}{|c|c|c|c|c|c|}
\hline AKAP12 & CST9L & GRSF1 & MEAF6 & PRKD3 & TAP1 \\
\hline AKR1A1 & CT45A6 & GSPT1 & MECOM & PROC & TASP1 \\
\hline AKR1C1 & СТА-246Н3.1 & GSTM2 & MEF2B & PROK1 & $\mathrm{TBCC}$ \\
\hline AKT1 & CTNNBL1 & GSTT1 & MEGF11 & PRPF31 & TBP \\
\hline AKT2 & CTSD & GTF2E1 & MEST & PRRX1 & TCF15 \\
\hline ALCAM & CUTA & GTF2F2 & METRN & PRSS16 & TCF7L2 \\
\hline ALDH5A1 & CX3CL1 & GTF2H5 & METTL13 & PRSS22 & TENM3 \\
\hline ALDH9A1 & CXCL1 & GTPBP4 & METTL4 & PRSS3 & TEX30 \\
\hline ALG5 & CXCL10 & GUCY1B3 & MFAP2 & PRSS36 & TFF1 \\
\hline ALMS1 & CXCL12 & GYG1 & MFSD7 & PSAT1 & TFF3 \\
\hline AMPD1 & CXCL13 & GYPC & MGMT & PSMB5 & TFPI2 \\
\hline ANKHD1 & CXCR4 & GZMB & MINOS1 & PSMB9 & TGFB1 \\
\hline ANKRD27 & CYB5B & GZMK & MKRN1 & PSMC4 & THBS4 \\
\hline ANXA3 & CYBRD1 & H2AFX & MLF2 & PSMD1 & TIAM1 \\
\hline ANXA4 & CYP27A1 & $\mathrm{H} 3 \mathrm{~F} 3 \mathrm{~A}$ & MLH1 & PSMD12 & TIMM10B \\
\hline $\mathrm{AOC} 1$ & CYP2E1 & HAP1 & MLX & PSMD14 & TIMM17B \\
\hline $\mathrm{AP} 2 \mathrm{~A} 2$ & CYP3A7 & $\mathrm{HBG} 2$ & MMP1 & PSME4 & TIMP1 \\
\hline APC & CYP4X1 & $\mathrm{HDAC1}$ & MMP10 & PTBP1 & TIMP2 \\
\hline API5 & CYP4Z1 & HDAC2 & MMP12 & PTCH2 & TIMP3 \\
\hline APOE & CYP51A1 & HECTD4 & MMP13 & PTEN & TKTL1 \\
\hline AQP10 & CYSTM1 & HES1 & MMP16 & PTGDS & TLE2 \\
\hline AQP5 & CYTH3 & HEY1 & MMP17 & PTGS2 & TM9SF2 \\
\hline AQP6 & D4S234E & HHIPL2 & MMP3 & PTP4A1 & TM9SF3 \\
\hline AQP9 & DAP & HIF1A & MMP7 & PTP4A2 & TMCC1 \\
\hline ARAF & DAPL1 & HIP1R & MMP9 & PTPRN2 & TMED5 \\
\hline ARAP1 & $\mathrm{DBI}$ & HIPK1 & MPZL1 & PTPRS & TMEM139 \\
\hline AREG & DCBLD2 & HIST1H1C & MRPL2 & PWP2 & TMEM14B \\
\hline ARFGEF2 & DCHS1 & HK2 & MRPL35 & QPRT & TMEM150A \\
\hline ARHGAP29 & DCK & HLAA & MRPL49 & R3HDM2 & TMEM161A \\
\hline ARHGDIA & DCTN5 & HLADMB & MRPS12 & RAB26 & TMEM259 \\
\hline ARL14 & DCTPP1 & HLADOB & MRPS17 & RAB27B & TMEM260 \\
\hline ARL6IP4 & DCUN1D4 & HMBOX1 & MRPS24 & RAB40B & TMEM45A \\
\hline ARMC1 & DCUN1D5 & HMGCS1 & MRPS9 & RAB5B & TMEM50A \\
\hline ARNT2 & DDB1 & HMGCS2 & MRS2 & RAB5C & TMPRSS3 \\
\hline ARPC4 & DDB2 & HMGN1 & $\mathrm{MSH} 2$ & RABIF & TMSB15B \\
\hline ASAP1 & DDR1 & HMOX2 & MSL1 & RAC1 & TMTC1 \\
\hline ASAP3 & DDX23 & HNRNPA1 & MSMO1 & RAC3 & $T M \times 2$ \\
\hline ASF1A & DDX49 & HNRNPUL2 & MST1 & RAD23A & TNFRSF17 \\
\hline ASIP & DEFB132 & HOPX & MT1G & RAD51 & TNS1 \\
\hline ASPA & DERL1 & HOXA5 & MTCP1 & RAD51AP1 & TOMM40 \\
\hline ASPHD1 & DFNB31 & HOXB6 & MTMR11 & RANBP1 & TONSL \\
\hline ASS1 & DHCR7 & HPN & MTMR2 & RANGAP1 & TOP1 \\
\hline ASUN & DHRS11 & HRASLS & MTPAP & RARRES2 & TOP2A \\
\hline ATM & DHRS9 & Hs.120332 & MTUS1 & RB1 & TOX3 \\
\hline ATP1B3 & DHX15 & HS3ST1 & MTX1 & RBBP7 & TP53 \\
\hline
\end{tabular}


Table 10 List of genes reported by studies included in this review (Continued)

\begin{tabular}{|c|c|c|c|c|c|}
\hline ATP5D & DHX29 & HS3ST5 & MUS81 & RBFA & TP53TG5 \\
\hline ATP5F1 & DIAPH3 & HSD11B2 & MUTYH & RBM11 & TP73 \\
\hline ATP5L & DICER1 & HSD17B11 & MXD1 & RBM39 & TPD52 \\
\hline ATP6V0E1 & DIRC1 & HSPA1L & MXI1 & $\mathrm{RCHY} 1$ & TPM2 \\
\hline ATP7B & DKK1 & HSPA4 & MYBPC1 & RER1 & TPP2 \\
\hline ATP8A2 & DLAT & HSPA8 & MYC & RFC3 & TPPP \\
\hline AUP1 & DLEU2 & HSPB7 & MYCBP & RGL2 & TPRKB \\
\hline AURKA & DLG1 & HSPD1 & MYL9 & RGP1 & TRA \\
\hline AURKC & DLG3 & HTATIP2 & MYO1D & RGS19 & TRAF3IP2 \\
\hline AVIL & DLGAP4 & HTN1 & MYOM1 & RHOT1 & TRAM1 \\
\hline B3GALNT1 & DLGAP5 & HTR3A & NANOS1 & RHPN2 & TRAPPC4 \\
\hline B3GNT2 & DMRT3 & ICAM1 & NASP & RIIAD1 & TRAPPC9 \\
\hline B4GALT5 & DNAH2 & ICAM5 & NBEA & RIN1 & TREML1 \\
\hline BAG3 & DNAH7 & ID1 & NBL1 & RIT1 & TREML2 \\
\hline BAIAP2L1 & DNAJB12 & ID4 & NBN & RNF10 & TRIAP1 \\
\hline BAK1 & DNAJB5 & IDI1 & NCAM1 & RNF13 & TRIM27 \\
\hline BASP1 & DNAJC16 & IFIT1 & NCAPD2 & RNF14 & TRIM49 \\
\hline BAX & DNASE1L3 & IGF1R & NCAPG & RNF148 & TRIM58 \\
\hline $\mathrm{BCHE}$ & DOCK3 & IGFBP2 & NCAPH & RNF34 & TRIML2 \\
\hline $\mathrm{BCL} 2 \mathrm{~A} 1$ & $\mathrm{DPH} 2$ & IGFBP5 & NCKAP5 & RNF6 & TRIT1 \\
\hline BCL2L11 & DPM1 & IGHM & NCOA1 & RNF7 & TRMT1L \\
\hline BCL2L12 & DPP7 & IGKC & NCOR2 & RNF8 & TRO \\
\hline BCR-ABL & DPYSL2 & IGKV1-5 & NCR2 & RNGTT & TRPV4 \\
\hline BEAN & DRD4 & $\mathrm{IHH}$ & NCSTN & RNPEPL1 & TRPV6 \\
\hline BEST4 & DTYMK & IKZF4 & NDRG2 & $\mathrm{ROBO} 1$ & TSPAN3 \\
\hline BFSP1 & DUSP2 & IL11RA & NDST1 & ROR1 & TSPAN4 \\
\hline BFSP2 & DUSP4 & IL15 & NDUFA12 & ROR2 & TSPAN6 \\
\hline BGN & DUX3 & IL17RB & NDUFA9 & RP13-347D8.3 & TSPAN7 \\
\hline BHLHE40 & DYNLT1 & IL1B & NDUFAB1 & RP13-36C9.6 & TSR1 \\
\hline BIN1 & DYRK3 & IL23A & NDUFAF4 & RPA3 & TाС31 \\
\hline BIRC5 & E2F2 & IL27 & NDUFB4 & RPL23 & TTLL6 \\
\hline BIRC6 & $\mathrm{ECH} 1$ & IL6 & NDUFS5 & RPL29P17 & TTPAL \\
\hline BLCAP & EDF1 & IL8 & NEBL & RPL31 & TYH1 \\
\hline BLMH & EDN1 & IMPA2 & NETO2 & RPL36 & TUBB3 \\
\hline BMP8B & EDNRA & ING3 & NEUROD2 & RPP30 & TUBB4A \\
\hline BMPR1A & EDNRB & INHBA & NFE2 & RPS15 & TUBB4Q \\
\hline BNIP3 & EEF1A2 & INPP5A & NFE2L3 & RPS16 & TUSC3 \\
\hline BOLA3 & EFCAB14 & INPP5B & NFIB & RPS19BP1 & UBD \\
\hline BPTF & EFEMP2 & INSR & NFKBIB & RPS24 & UBE2I \\
\hline BRCA1 & EFNB2 & INTS12 & NFS1 & RPS28 & UBE2K \\
\hline BRCA2 & EGF & INTS9 & NID1 & RPS4Y1 & UBE2L3 \\
\hline BRSK1 & EGFR & IRF2BP1 & NIT1 & RPS6KA2 & UBE4B \\
\hline BTN3A3 & EHD1 & $|\mathrm{SCA}|$ & NKIRAS2 & RPSA & UBR5 \\
\hline BTNL9 & EHF & ISG20 & NKX31 & RRAGC & UGT2B17 \\
\hline C11orf16 & El24 & ITGAE & NKX62 & RRBP1 & UGT8 \\
\hline
\end{tabular}


Table 10 List of genes reported by studies included in this review (Continued)

\begin{tabular}{|c|c|c|c|c|c|}
\hline C11orf74 & EIF1 & ITGB2 & NLGN1 & RRN3 & UHRF1BP1 \\
\hline C12orf5 & EIF2AK2 & ITGB6 & NOP5/58 & RSL24D1 & UMOD \\
\hline C16orf89 & EIF3K & ITGB7 & NOS3 & RSU1 & UPK1A \\
\hline C17orf45 & EIF4E2 & ITLN1 & $\mathrm{NOTCH} 4$ & RTN4R & UPK1B \\
\hline C17orf53 & EIF5 & ITM2A & NOV & $\mathrm{RXRB}$ & UQCRC2 \\
\hline C17orf70 & ELF3 & ITM2C & NOX1 & RYBP & URI1 \\
\hline C1orf109 & ELF5 & ITPR2 & NPAS3 & RYR3 & USP14 \\
\hline C1orf115 & EML4 & ITPRIP & NPR1 & S100A10 & USP18 \\
\hline C1orf159 & ENC1 & JAG2 & NPR3 & S100A4 & USP21 \\
\hline C1orf198 & ENOPH1 & JAK2 & NPTX2 & S100P & UST \\
\hline C1orf27 & ENSA & JAKMIP2 & NPTXR & SAMD4B & UTP11L \\
\hline C1orf68 & ENTPD4 & KCNB1 & NPY & SASH1 & UTP20 \\
\hline C1QTNF3 & EPB41L4A & KCNE3 & NRBP2 & SCAMP3 & UVRAG \\
\hline C20orf199 & EPCAM & $\mathrm{KCNH} 2$ & NRG4 & SCARF1 & VDR \\
\hline C2orf72 & EPHB2 & KCNJ16 & NRP1 & SCG2 & VEGFA \\
\hline C4A & EPHB3 & KCNN1 & NSFL1C & SCGB1C1 & VEGFB \\
\hline C4BPA & EPHB4 & KCNN3 & NSL1 & SCGB3A1 & VEZF1 \\
\hline C6orf120 & EPOR & KCTD1 & NSMCE4A & SCNM1 & VPS39 \\
\hline C6orf124 & ERBB3 & KCTD5 & NT5C3A & $\mathrm{SCO} 2$ & VPS52 \\
\hline C9orf3 & ERCC8 & KDELC1 & NTAN1 & SCUBE2 & VPS72 \\
\hline C9orf47 & ERMP1 & KDELR1 & NTF4 & SDF2L1 & VTCN1 \\
\hline CA13 & ESF1 & KDELR2 & NUDT21 & SEC14L2 & VTI1B \\
\hline CACNA1B & ESM1 & KDM4A & NUDT9 & SELT & WBP2 \\
\hline CACNG6 & ESR1 & Ki67 & NUS1 & SEMA3A & WBP4 \\
\hline CADM1 & ESRP2 & KIAA0125 & OAS3 & SENP3 & WDR12 \\
\hline CALML3 & ESYT1 & KIAA0141 & OASL & SENP6 & WDR45B \\
\hline CAMK2B & ETS1 & KIAA0226 & ODF4 & SEPN1 & WDR7 \\
\hline CAMK2N1 & ETV1 & KIAA0368 & OGFOD3 & SERPINB6 & WDR77 \\
\hline CANX & EVA1A & KIAA1009 & OGN & SERPIND1 & WIT1 \\
\hline CAP1 & EXOC6B & KIAA1033 & OPA3 & SERPINF1 & WIZ \\
\hline CAP2 & EXTL1 & KIAA1324 & OR10A3 & SERTAD4 & WNK4 \\
\hline CAPN13 & EYA2 & KIAA1551 & OR2AG1 & SETBP1 & WNT16 \\
\hline CAPN5 & $\mathrm{F} 2 \mathrm{R}$ & KIAA2022 & OR4C15 & SF3A3 & WT1 \\
\hline CASC3 & FAAH & KIAA4146 & OR51B5 & SF3B4 & WTAP \\
\hline CASP9 & FABP1 & KIF3A & OR5111 & SGCB & WWOX \\
\hline CASS4 & FABP7 & $\mathrm{KIFC3}$ & OR6F1 & SGCG & XBP1 \\
\hline CATSPERD & FADS1 & $\mathrm{KIT}$ & OR9G9 & SGPP1 & XPA \\
\hline CC2D1A & FADS2 & KLF12 & OSGEPL1 & SH3PXD2A & XPO4 \\
\hline CCBL1 & FAM133A & KLF5 & OSGIN2 & SHFM1 & XYLT1 \\
\hline CCDC130 & FAM135A & KLHDC3 & OSM & SHOX & Y09846 \\
\hline CCDC135 & FAM155B & KLHL7 & OXTR & SIDT1 & YBX1 \\
\hline CCDC147 & FAM174B & KLK10 & $\mathrm{P} 2 \mathrm{RX} 4$ & SIGLEC8 & YIPF3 \\
\hline CCDC167 & FAM19A4 & KLK6 & PABPC4 & SIRT5 & YIPF6 \\
\hline CCDC19 & FAM211B & KPNA3 & PAGR1 & SIRT6 & YLPM1 \\
\hline CCDC53 & FAM217B & KPNA6 & PAH & SIVA1 & YWHAE \\
\hline
\end{tabular}


Table 10 List of genes reported by studies included in this review (Continued)

\begin{tabular}{|c|c|c|c|c|c|}
\hline CCDC9 & FAM49B & KRT10 & PAK4 & SIX2 & YWHAZ \\
\hline CCL13 & FAM8A1 & KRT12 & PALB2 & SKA3 & ZBTB11 \\
\hline CCL2 & FANCB & KYNU & PARD6B & SLAMF7 & ZBTB16 \\
\hline CCL28 & FANCE & L1TD1 & PAX6 & SLC12A2 & ZBTB8A \\
\hline CCM2L & FANCF & LAMB1 & PBK & SLC12A4 & ZC3H13 \\
\hline CCNA2 & FANCG & LAMTOR5 & PBX2 & SLC14A1 & ZCCHC8 \\
\hline CCNG2 & FANCI & LARP4 & PBXIP1 & SLC15A2 & ZEB2 \\
\hline CCT6А & FARP1 & LAX1 & PCF11 & SLC1A1 & ZFHX4 \\
\hline CCZ1 & FAS & LAYN & PCGF3 & SLC1A3 & ZFP91 \\
\hline CD34 & FASLG & LBR & PCK1 & SLC22A5 & ZFR2 \\
\hline CD38 & FBXL18 & LCMT2 & PCNA & SLC25A37 & ZKSCAN7 \\
\hline CD44 & FCGBP & LCTL & PCNXL2 & SLC25A41 & ZMYND11 \\
\hline CD46 & FCGR3B & LDB1 & PCOLCE & SLC25A5 & ZNF106 \\
\hline CD70 & FEN1 & LDHB & PCSK6 & SLC26A9 & ZNF12 \\
\hline CD97 & FEZ1 & LGALS4 & PDCD2 & SLC27A6 & ZNF124 \\
\hline CDC42EP4 & FGF2 & LGR5 & PDE3A & SLC29A1 & ZNF148 \\
\hline CDCA2 & FGFBP1 & LHB & PDGFA & SLC2A1 & ZNF155 \\
\hline $\mathrm{CDH} 12$ & FGFR1OP & LHX1 & PDGFRA & SLC2A5 & ZNF180 \\
\hline $\mathrm{CDH} 19$ & FGFR1OP2 & LIN28A & PDGFRB & SLC37A4 & ZNF200 \\
\hline $\mathrm{CDH} 3$ & FGFR2 & LINGO1 & PDP1 & SLC39A2 & ZNF292 \\
\hline $\mathrm{CDH} 4$ & FHL2 & LIPA & PDSS1 & SLC4A11 & ZNF337 \\
\hline $\mathrm{CDH} 5$ & FILIP1 & LIPC & PDZK1 & SLC5A1 & ZNF432 \\
\hline CDK17 & FJX1 & LIPG & PEBP1 & SLC5A3 & ZNF467 \\
\hline CDK20 & FKBP11 & LMO3 & PEX11A & SLC5A5 & ZNF48 \\
\hline CDK5R1 & FKBP1B & LMO4 & PEX6 & SLC6A3 & ZNF503 \\
\hline CDK8 & FKBP7 & LOC100129250 & PFAS & SLC7A2 & ZNF521 \\
\hline CDKN1A & FLII & LOC149018 & PGAM1 & SMAD2 & ZNF569 \\
\hline CDY1 & FLJ41501 & LOC1720 & PHF3 & SMC4 & ZNF644 \\
\hline CDYL2 & FLNC & LOC389677 & $\mathrm{PHGDH}$ & SMG1 & ZNF71 \\
\hline CEACAM5 & FLOT2 & LOC642236 & PHKA1 & SMPD2 & ZNF711 \\
\hline CEACAM6 & FLT1 & LOC646808 & PHKA2 & SNIP1 & ZNF74 \\
\hline CEACAM7 & FMN2 & LOC90925 & $\mathrm{PI3}$ & SNRPA1 & ZNF76 \\
\hline CEP55 & FMO1 & LPAR6 & PIC3CD & SNRPC & ZNF780B \\
\hline CES1 & FN1 & LPCAT2 & PIGC & SNRPD3 & ZYG11A \\
\hline CES2 & FOXA2 & LPCAT4 & PIGR & SNX13 & \\
\hline $\mathrm{CFI}$ & FOXD4L2 & LPHN2 & PIK3CG & SNX19 & \\
\hline $\mathrm{CH} 25 \mathrm{H}$ & FOXJ1 & LRIG1 & PIP5K1B & SNX7 & \\
\hline CHIT1 & FOXO3 & LRIT1 & PITRM1 & SOAT2 & \\
\hline
\end{tabular}

Gene names have been standardised. Genes in bold were selected by more than two studies.

Seidal [35], Schlumbrecht et al. [40], Yoshihara et al. [43], Denkert et al. [45], Hartmann et al. [57], Iba et al. [60], and Kamazawa et al. [61]. Studies falling into the other treatments group were Obermayr et al. [27], Sabatier et al. [27], Yan et al. [42], Netinatsunthorn et al. [51], and Helleman et al. [53]. The results of the gene set enrichment using the KEGG system may be seen in Figures 2 and 3. From the plots, it may be seen that both groups identify several cancer-related pathways relevant to the drug mechanisms of action. 
Table 11 Genes chosen most commonly by studies in review

\begin{tabular}{|c|c|c|c|}
\hline Gene symbol & Number of studies & Function & Expression links to cancer in literature \\
\hline AGR2 & 4 & Cell migration and growth & Prostate, breast, ovarian, pancreatic \\
\hline MUTYH & 3 & Oxidative DNA damage repair & Colorectal \\
\hline AKAP12 & 3 & Subcellular compartmentation of PKA & Colorectal, lung, prostate \\
\hline TP53 & 3 & Cell cycle regulation & Breast \\
\hline TOP2A & 3 & Required for DNA replication & Breast, prostate, ovarian \\
\hline FOXA2 & 3 & Liver-specific transcription factor & Lung, prostate \\
\hline SRC & 2 & Regulation of cell growth & Colon, liver, lung, breast, pancreatic \\
\hline SIVA1 & 2 & Pro-apoptotic protein & Many cancers \\
\hline ALDH9A1 & 2 & Aldehyde dehydrogenase & Many cancers \\
\hline LGR5 & 2 & Associated with stem cells & Cancer stem cells \\
\hline EHF & 2 & Epithelial differentiation and proliferation & Prostate \\
\hline BAX & 2 & Apoptotic activator & Colon, breast, prostate, gastric, leukaemia \\
\hline CES2 & 2 & Intestine drug clearance & Colorectal \\
\hline CPE & 2 & Synthesis of hormones and neurotransmitters & \\
\hline FGFBP1 & 2 & Cell proliferation, differentiation and migration & Colorectal, pancreatic \\
\hline TUBB4A & 2 & Component of microtubules & \\
\hline ZNF12 & 2 & Transcription regulation & \\
\hline RBM39 & 2 & Steroid hormone receptor-mediated transcription & \\
\hline RFC3 & 2 & Required for DNA replication & \\
\hline GNPDA1 & 2 & Triggers calcium oscillations in mammalian eggs & \\
\hline ANXA3 & 2 & Regulation of cellular growth & Prostate, ovarian \\
\hline NFIB & 2 & Activates transcription and replication & Breast \\
\hline ACTR3B & 2 & Actin cyctoskeleton organisation & Lung \\
\hline YWHAE & 2 & Mediates signal transduction & Lung, endometrial \\
\hline CYP51A1 & 2 & Drug metabolism and lipid synthesis & \\
\hline HMGCS1 & 2 & Cholesterol synthesis and ketogenesis & \\
\hline ZMYND11 & 2 & Transcriptional repressor & \\
\hline FADS2 & 2 & Regulates unsaturation of fatty acids & \\
\hline SNX7 & 2 & Family involved in intracellular trafficking & \\
\hline ARHGDIA & 2 & Regulates the GDP/GTP exchange reaction of the Rho proteins & Prostate, lung, \\
\hline NDST1 & 2 & Inflammatory response & Prostate, breast \\
\hline $\mathrm{AOC1}$ & 2 & Catalyses degredation of such as histamine and spermidine & \\
\hline DAP & 2 & Positive mediator of programmed cell death & \\
\hline ERCC8 & 2 & Transcription-coupled nucleotide excision repair & \\
\hline GUCY1B3 & 2 & Catalyzes conversion of GTP to the second messenger cGMP & \\
\hline HDAC1 & 2 & Control of cell proliferation and differentiation & Prostate, breast, colorectal, gastric \\
\hline HDAC2 & 2 & Transcriptional regulation and cell cycle progression & Cervical, gastric, colorectal \\
\hline IGFBP5 & 2 & Cell proliferation, differentiation, survival, and motility & Breast \\
\hline IL6 & 2 & Transcriptional inflammatory response, B cell maturation & Many cancers \\
\hline LSAMP & 2 & Neuronal surface glycoprotein & Osteosarcoma \\
\hline MDK & 2 & Cell growth, migration, angiogenesis & Many cancers \\
\hline MYCBP & 2 & Stimulates the activation of E box-dependent transcription & \\
\hline S100A10 & 2 & Transport of neurotransmitters & Colorectal, lung, breast \\
\hline
\end{tabular}


Table 11 Genes chosen most commonly by studies in review (Continued)

\begin{tabular}{|c|c|c|c|}
\hline SLC1A3 & 2 & Glutamate transporter & \\
\hline NCOA1 & 2 & Stimulates hormone-dependent transcription & Breast, prostate \\
\hline TIAM1 & 2 & Modulates the activity of Rho GTP-binding proteins & Many cancers \\
\hline VEGFA & 2 & Angiogenesis, cell growth, cell migration, apoptosis & Many cancers \\
\hline RPL36 & 2 & Component of ribosomal 605 subunit & \\
\hline LBR & 2 & Anchors lamina and heterochromatin to the nuclear membrane & \\
\hline$A B C B 1$ & 2 & ATP-dependent drug efflux pump for xenobiotic compounds & Many cancers \\
\hline FASLG & 2 & Required for triggering apoptosis in some cell types & Many cancers \\
\hline TIMP1 & 2 & Extracellular matrix, proliferation, apoptosis & Many cancers \\
\hline FN1 & 2 & Cell adhesion, motility, migration processes & Many cancers \\
\hline TGFB1 & 2 & Proliferation, differentiation, adhesion, migration & Prostate, breast, colon, lung, bladder \\
\hline XPA & 2 & DNA excision repair & Many cancers \\
\hline ABCB10 & 2 & Mitochondrial ATP-binding cassette transporter & \\
\hline POLH & 2 & Polymerase capable of replicating UV-damaged DNA for repair & \\
\hline ITGAE & 2 & Adhesion, intestinal intraepithelial lymphocyte activation & \\
\hline ZNF200 & 2 & Zinc finger protein & \\
\hline COL3A1 & 2 & Collagen type III, occurring in most soft connective tissues & \\
\hline ACKR3 & 2 & G-protein coupled receptor & \\
\hline EPHB3 & 2 & Mediates developmental processes & Lung, colorectal \\
\hline NBN & 2 & Double-strand DNA repair, cell cycle control & \\
\hline PCF11 & 2 & May be involved in Pol II release following polymerisation & \\
\hline DFNB31 & 2 & Sterocilia elongation, actin cystoskeletal assembly & \\
\hline BRCA2 & 2 & Double-strand DNA repair & Breast, ovarian \\
\hline AADAC & 2 & Arylacetamide deacetylase & \\
\hline CD38 & 2 & Glucose-induced insulin secretion & Leukaemia \\
\hline CHIT1 & 2 & Involved in degradation of chitin-containing pathogens & \\
\hline CXCR4 & 2 & Receptor specific for stromal-derived-factor-1 & Breast, glioma, kidney, prostate \\
\hline EFNB2 & 2 & Mediates developmental processes & \\
\hline MECOM & 2 & Apoptosis, development, cell differentiation, proliferation & Leukaemia \\
\hline FILIP1 & 2 & Controls neocortical cell migration & Ovarian \\
\hline HSPB7 & 2 & Heat shock protein & \\
\hline LRIG1 & 2 & Regulator of signaling by receptor tyrosine kinases & Glioma \\
\hline MMP1 & 2 & Breakdown of extracellular matrix & Gastric, breast \\
\hline PSAT1 & 2 & Phosphoserine aminotransferase & \\
\hline SDF2L1 & 2 & Part of endoplasmic reticulum chaperone complex & \\
\hline TCF15 & 2 & Regulation of patterning of the mesoderm & \\
\hline EPHB2 & 2 & Contact-dependent bidirectional signaling between cells & Colorectal \\
\hline ETS1 & 2 & Involved in stem cell development, cell senescence and death & Many cancers \\
\hline TRIM27 & 2 & Male germ cell differentiation & Ovarian, endometrial, prostate \\
\hline MARK4 & 2 & Mitosis, cell cycle control & Glioma \\
\hline B4GALT5 & 2 & Biosynthesis of glycoconjugates and saccharides & \\
\hline
\end{tabular}

Genes listed by number of papers selecting each gene. Gene function and links to cancer obtained via cursory literature search.

It is informative to consider the KEGG terms in the context of the mechanisms of action of the chemotherapy drugs applied. Both groups contain patients treated with platinum single agents or platinum-containing combinations. It should therefore be expected that processes associated with the mechanism of action of 


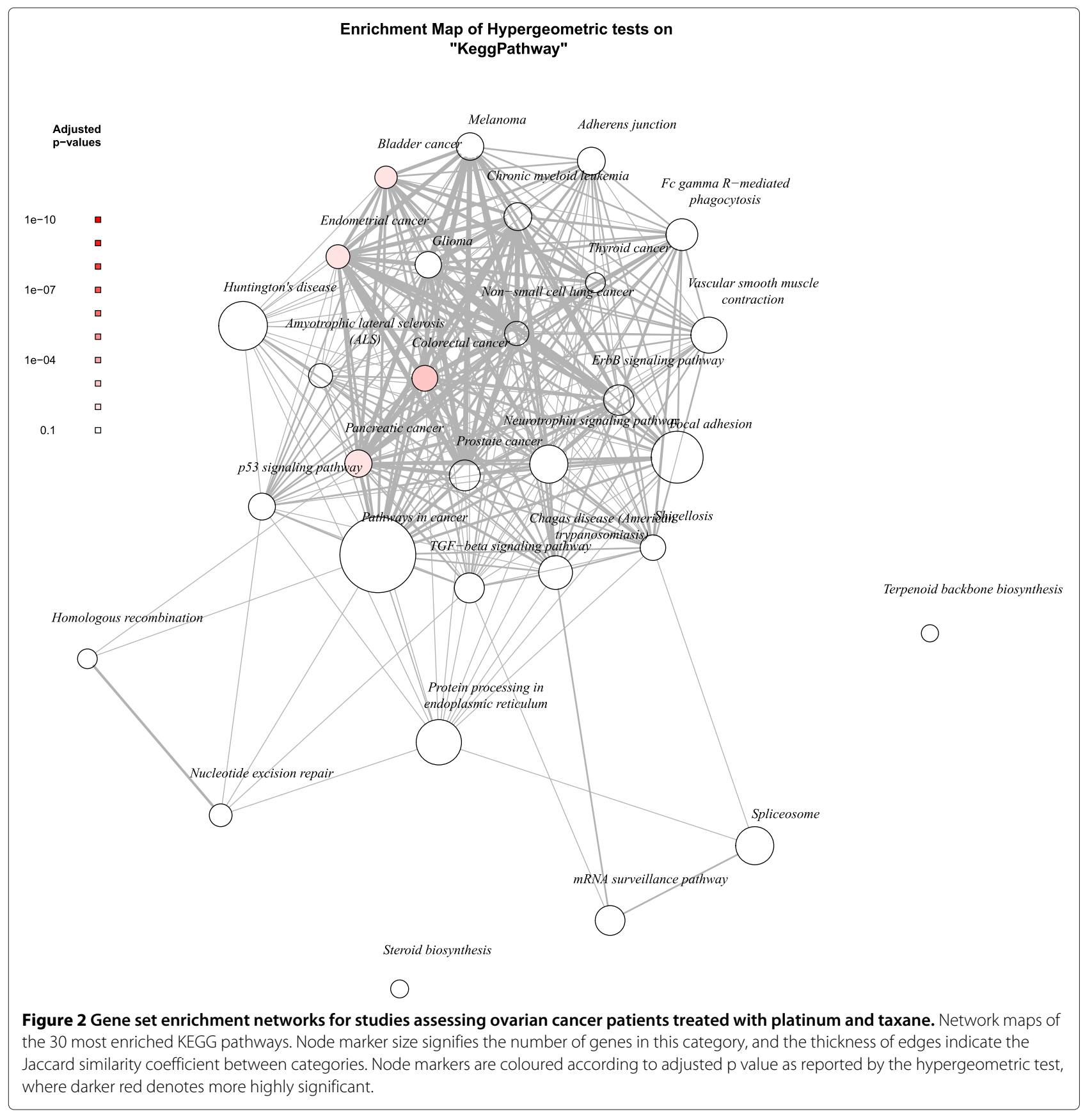

platinum will be enriched. Once activated, the platinum binds to DNA and results in the formation of monoadducts, intra-strand crosslinking, inter-strand crosslinking and protein crosslinking. This DNA structure change affects the ability of the DNA to be unwound and replicated, resulting in the triggering of the G2M DNA damage checkpoint and cell cycle arrest. The affected cell will attempt DNA repair and, if unsuccessful, undergo apoptosis [69]. Expected KEGG terms therefore include those relating to apoptosis and DNA damage.
From Figure 2, KEGG pathways highlighted for this group of studies include ten cancer-specific terms and six cancer-related terms. Here italics denote a KEGG term. The ErbB signalling pathway has been found to influence in proliferation, migration, differentiation and apoptosis in cancer [70] and overexpression of ERBB1 and ERBB2 have been implicated in head and neck and breast cancers. The neurotrophin signalling pathway is known to trigger MAPK and PI3K signalling, affecting differentiation, proliferation and development, and survival, growth, motility and angiogenesis respectively [71]. Altered expression of 


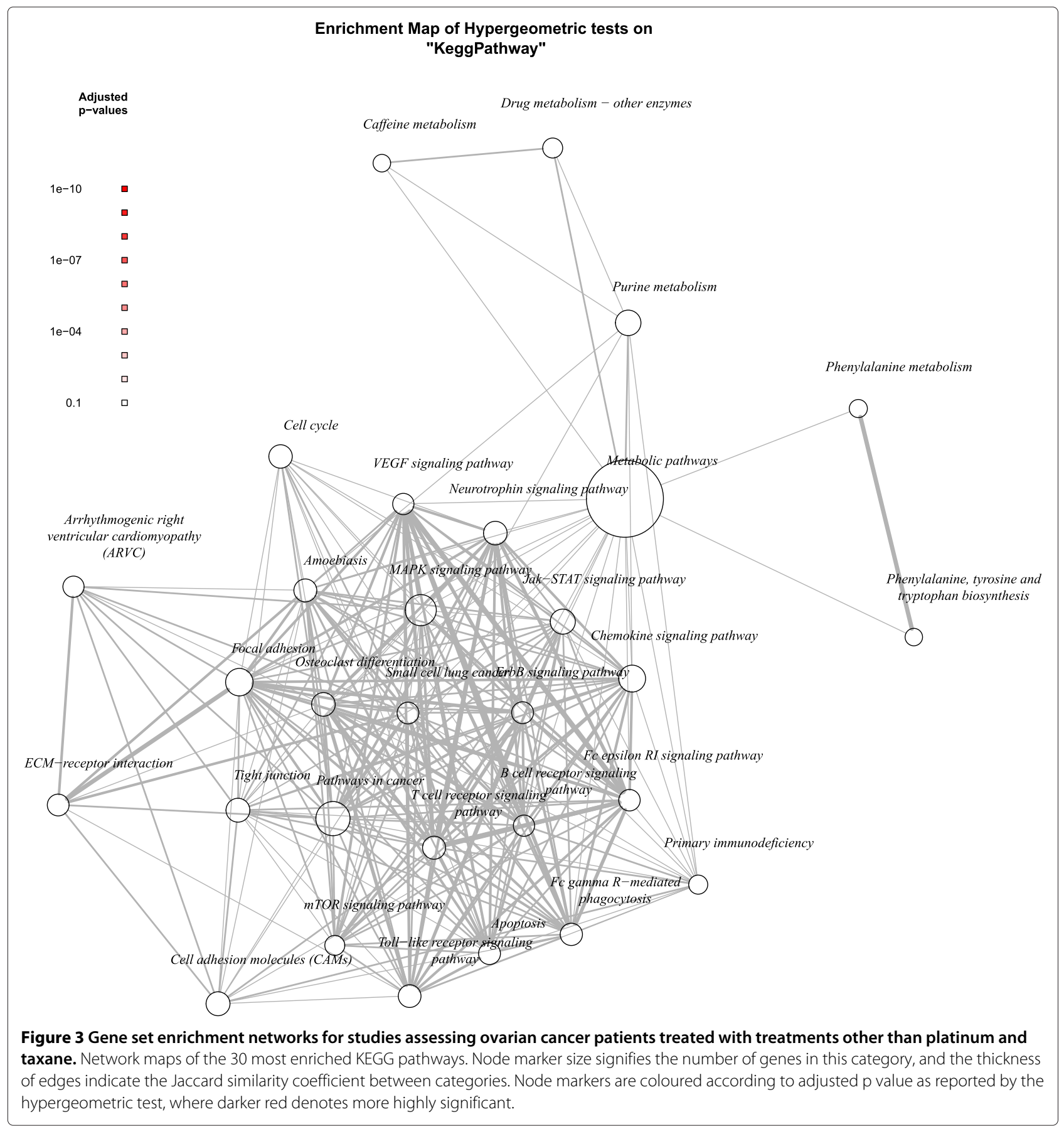

genes in this pathway has been found to correlate with poorer survival in colon, breast, lung and prostate cancers. Changes in expression of genes relating to focal adhesion, which is responsible for attachment of cells to the extracellular matrix, have been implicated in cancer migration, invasion, survival and growth [72]. The TGF-beta signalling pathway also regulates many cellular processes, including proliferation, cellular adhesion and motility, coregulation of telomerase function, regulation of apoptosis, angiogenesis, immunosuppression and DNA repair [73]. The p53 signalling pathway has many varied links to cancer. This pathway many be triggered by various stress signals and can result in several responses, including cell cycle arrest, apoptosis, the inhibition of angiogenesis and metastasis, and DNA repair [74]. Finally, nucleotide excision repair is known to promote cancer development when both up and down regulated. Downregulation correlates is thought to increases susceptibility 
to mutation formation and hence the formation of cancer [75], whereas up-regulation has been found to correlate with resistance to platinum as the DNA damage caused by the chemotherapy agent is repaired [76].

The first group of studies considered patients treated with taxanes in addition to platinum. Taxanes act by stabilising tubulin, preventing the microtubule structure formation required for mitosis. This results in cell cycle arrest at the G2/M DNA damage checkpoint and apoptosis. Mechanisms for taxane resistance are, however, not well understood. Two suggested mechanisms include the increased expression of multidrug transporters, and changes in the expression of the $\beta$-tubulin isoforms [77]. Neither of these mechanisms seem to be enriched in the platinum and taxol group. In addition to the single-agent effects of platinum and taxanes, there is an additional synergistic effect [78]. However, this effect is also not well studied and hence the mechanisms by which this occurs are not clear.

The second group, as seen in Figure 3, was composed of studies applying chemotherapy treatments other than platinum and taxanes. This group is heterogeneous with respect to chemotherapy treatment, and mainly consists of studies reporting treatment as 'platinum-based. The other drug explicitly mentioned by studies in this group is cyclophosphamide. This drug is an alkylating agent and acts to form adducts in DNA [79]. This DNA damage triggers the G2/M DNA damage checkpoint, resulting in DNA repair or apoptosis. This suggests that the same DNA repair mechanisms related to platinum treatment are also relevant to cyclophosphamide. For this group, the KEGG pathway analysis shows that the gene set is enriched with 14 pathways related to cancer, in addition to two general cancer-related terms. The mTOR signalling pathway is downstream to the PI3K/AKT pathway and regulates growth, proliferation and survival [80]. The MAPK signalling pathway controls the cell cycle, and has been found to contribute to the control of proliferation, differentiation, apoptosis, migration and inflammation in cancer [81]. The chemokine signalling pathway has been found to regulate growth, survival and migration in addition to its role in inflammation [82]. Angiogenesis and vasculogenesis are known to be regulated by the VEGF signalling pathway [83], which is already the target of treatments such as bevacizumab. Purine metabolism is required for the production and recycling of adenine and guanine, and hence is required for DNA replication. This process is the target of chemotherapies such as methotrexate. The term drug metabolism - other enzymes is partially cancer related; this term refers to five drugs: azathioprine, 6-mercaptopurine, irinotecan, fluorouracil and isoniazid. Of these, two are chemotherapy treatments; irinotecan is a topoisomerase-I inhibitor and fluorouracil acts as a purine analogue. Also featuring in Figure 3 are apoptosis, ErbB signalling pathway, focal adhesion, neurotrophin signalling pathway, B cell receptor signalling pathway and Jak-STAT signalling pathway, all of which are known to be related to cancer.

Overall, the gene sets appear to be enriched for cancerrelated resistance mechanisms [84]. However, when combined there is little evidence from this analysis to suggest that the signatures are capturing chemotherapy-specific mechanisms in addition to more general survival pathways. The DNA repair terms may suggest a response to platinum-based treatment, though the down-regulation of these mechanisms is also related to cancer development and resistance in general [85]. It is likely that, due to the varying reliability suggested by the bias analysis and the reported model development techniques, the signalto-noise ratio of informative genes is low when the gene signatures are combined, preventing the identification of processes of interest.

\section{Model predictive ability \\ Sensitivity and specificity}

The comparison of the success of the various models is difficult, particularly due to the fact that many papers report different metrics as measures of model accuracy. Many of these are also incomplete, not providing enough information to fully describe the model. Ideally, models should be applied to an independent set of samples with known outcomes and performance measures on this data set reported. For classification models an informative set of measures would be positive predictive value, negative predictive value, specificity and sensitivity:

$$
\begin{aligned}
\text { Sensitivity } & =\frac{n_{\text {true positive }}}{n_{\text {true positive }}+n_{\text {false negative }}} \\
\text { Specificity } & =\frac{n_{\text {true negative }}}{n_{\text {true negative }}+n_{\text {false positive }}} \\
\text { PPV } & =\frac{n_{\text {true positive }}}{n_{\text {true positive }}+n_{\text {false positive }}} \\
\text { NPV } & =\frac{n_{\text {true negative }}}{n_{\text {true negative }}+n_{\text {false negative }}}
\end{aligned}
$$

where $n_{\text {true positive }}$ is the number of true positive predictions, $n_{\text {false positive }}$ is the number of false positive predic-

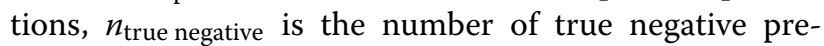
dictions and $n_{\text {false negative }}$ is the number of false negative predictions.

Together these provide information on true positive and negative rates as well as false positive and false negative rates, all of which are important when assessing the performance of a model.

Using the sensitivity and specificity the positive and negative likelihood ratios may be calculated and, using the prevalence of the condition in the test population, the 
probability of a patient having the condition based on the test results may be found, as in the equations below.

$$
\begin{aligned}
\mathrm{LR}_{+\mathrm{ve}} & =\frac{\text { sensitivity }}{1-\text { specificity }} \\
\mathrm{LR}_{-\mathrm{ve}} & =\frac{1-\text { sensitivity }}{\text { specificity }} \\
P(\text { Condition }+\mid \text { Test }+) & =\frac{\frac{P(\text { Condition }+)}{1-P(\text { Condition }+)} \cdot \mathrm{LR}_{+\mathrm{ve}}}{\frac{P(\text { Condition }+)}{1-P(\text { Condition }+)} \cdot \mathrm{LR}_{+\mathrm{ve}}+1} \\
P(\text { Condition }+\mid \text { Test }-) & =\frac{\frac{P(\text { Condition }-)}{1-P(\text { Condition }-)} \cdot \mathrm{LR}_{-\mathrm{ve}}}{\frac{P(\text { Condition }-)}{1-P(\text { Condition }-)} \cdot \mathrm{LR}_{-\mathrm{ve}}+1}
\end{aligned}
$$

These post-test probabilities are much easier to interpret and incorporate the prevalence of the condition. It should be noted that in order for the test to be applied in a clinical situation the pre-test probabilities used, $P($ Condition + ) and $P($ Condition -$)$, should be correct for the population of patients to whom the test will be applied. Here the sample prevalence from each study was used for convenience. However, it would be informative to recalculate $P($ Condition $+\mid$ Test +$)$ and $P($ Condition $+\mid$ Test -$)$ for the general population of ovarian cancer patients, as this would provide a better comparison between models.

Table 12 details the post-test probabilities of patients having a condition based on a positive or negative test result from the models developed by studies in this review. The papers appearing here are those that supplied sensitivity and specificity and the numbers of patients with and with without the condition, or alternative information allowing these to be calculated such as numbers of true and false positives and negatives.

From the table it may be seen that there is a great variety between the success of the models. For example, Kamazawa et al. [61] and Hartmann et al. [57] both achieved $P$ (Condition $+\mid$ Test +$)=0.95$ on their respective samples of the population. This means that if a patient tests positive, there is a $95 \%$ probability that they are positive for the condition in question, which in these cases are 'responding to chemotherapy' and 'poor prognosis' respectively. In contrast, Obermayr et al. [27], Helleman et al. [53] and Gevaert et al. [49] only achieved $P($ Condition $+\mid$ Test + ) of between 0.20 and 0.40 . These results suggest that the tests are not able to predict the outcome of a patient any better than a random choice, and in the case of tests in the region of 0.20 it is likely that most patients are simply assigned to the same class.

The ability of tests to not commit type II errors and give false negatives is also important. Ferriss et al. [33] and Hartmann et al. [57] both achieved well in this regard, with $P($ Condition $+\mid$ Test -$)=0.07$ and $P($ Condition + |Test -$)=0.05$ respectively. Several studies, by contrast, had very poor probabilities of false negatives; Obermayr et al. [27], Helleman et al. [53] and Gevaert et al. [49] all have
$P($ Condition $+\mid$ Test -$)>0.5$, which suggests that these models give a false negative more often than a random assignment.

Kamazawa et al. [61] and Selvanayagam et al. [59] both achieved extremely impressive prediction abilities, as may be seen by the very large $P($ Condition $+\mid$ Test +$)$ and very small $P$ (Condition $+\mid$ Test - ) values. However, these studies exemplify why care must be taken in assessing the predictive ability of models. Both studies calculated sensitivity and specificity based on only training set results and hence there is no way to judge the generalisability of the models. There is a tendency for models to perform better on the training set than any following independent data set to which it is subsequently applied. Secondly, the training set used by Selvanayagam et al. [59] is extremely small at eight patients and has a 50:50 ratio of chemoresistant to chemosensitive patients. This sample is not representative of the population and hence the values of $P($ Condition $+\mid$ Test +$)$ and $P($ Condition $+\mid$ Test -$)$ will be skewed by unrepresentative $P($ Condition +$)$ and $P$ (Condition-).

Overall, the most successful model of this group is that by Hartmann et al. [57] as it makes predictions with good reliability and has been validated on an independent data set. The least successful models were Obermayr et al. [27], Helleman et al. [53] and Gevaert et al. [49]. These studies suffered from low ability to identify true positives and high probability of false positives, resulting in poor predictive ability.

\section{Hazard ratios}

It is common for studies of survival to quote hazard ratios comparing the results of clusters identified by classification models or relative-risk models such as Cox proportional hazards regression. These ratios represent the ratio of the probability of an event occurring to a patient in each of the two groups. The event is often death, but could also be recurrence for example. The studies listed in Table 13 supplied hazard ratios as measures of predictive ability. The hazard ratios vary from 0.23 to 4.6 with the majority around 2 to 3 . A hazard ratio that is not equal to 1 suggests that the variable has predictive ability, and a ratio of 4 , for example, suggests that a member of the high-risk group is 4 times as likely to die within the study period than a member of the low-risk group. The study with the highest hazard ratio is Spentzos et al. [58], with $\mathrm{HR}=4.6$. This is closely followed by Raspollini [56] with $\mathrm{HR}=0.23$ and Skirnisdottir and Seidal [35] with $\mathrm{HR}=4.12$. The confidence intervals on the hazard ratios of all the studies are large and, with the exception of Spentzos et al. [58], at the lowest edge the hazard ratio is very close to 1 . This suggests that, although all these hazard ratios were found to be significant, some were close to not reaching the arbitrary $5 \%$ level. Most notable are Roque 
Table 12 Prediction metrics for studies reporting sensitivity and specificity

\begin{tabular}{|c|c|c|c|c|c|c|c|c|c|}
\hline Study & Prediction & Sensitivity & Specificity & $\mathrm{LR}_{+\mathrm{ve}}{ }^{\dagger}$ & $\mathrm{LR}_{\text {-ve }}{ }^{\dagger}$ & $P(\mathrm{C}+)^{\dagger}$ & $P(\mathrm{C}-)^{\dagger}$ & $P(\mathrm{C}+\mid \mathrm{T}+)^{\dagger}$ & $P(\mathrm{C}+\mid \mathrm{T}-)$ \\
\hline Li et al. [3] & Chemoresistance & $0.96^{*}$ & $0.23^{*}$ & 1.24 & 0.18 & $\frac{22}{44}$ & $\frac{22}{44}$ & 0.55 & 0.15 \\
\hline Obermayr et al. [27] & RFS & $0.22^{*}$ & $0.85^{*}$ & 1.47 & 0.92 & $\frac{46}{216}$ & $\frac{170}{216}$ & 0.28 & 0.77 \\
\hline Ferriss et al. [33] & Chemoresponse & $0.94^{*}$ & $0.29^{*}$ & 1.33 & 0.20 & $\frac{85}{119}$ & $\frac{34}{119}$ & 0.77 & 0.07 \\
\hline Sabatier et al. [37] & Prognosis & $0.62^{*}$ & $0.62^{*}$ & 1.64 & 0.62 & $\frac{194}{366}$ & $\frac{172}{366}$ & 0.65 & 0.35 \\
\hline Yoshihara et al. [43] & PFS & $0.64^{*}$ & $0.69^{*}$ & 2.06 & 0.52 & $\frac{45}{87}$ & $\frac{39}{87}$ & 0.69 & 0.30 \\
\hline Williams et al. [44] & Prognosis & $0.77^{*}$ & $0.56^{*}$ & 1.75 & 0.41 & $\frac{97}{143}$ & $\frac{46}{143}$ & 0.79 & 0.16 \\
\hline Gevaert et al. [49] & Chemoresistance & $0.67^{*}$ & $0.40^{*}$ & 1.12 & 0.82 & $\frac{15}{45}$ & $\frac{30}{45}$ & 0.36 & 0.62 \\
\hline Helleman et al. [53] & Chemoresistance & $0.89^{*}$ & $0.56^{*}$ & 2.02 & 0.20 & $\frac{9}{72}$ & $\frac{63}{72}$ & 0.22 & 0.58 \\
\hline De Smet et al. [52] & Chemoresistance & $0.71^{\dagger}$ & $0.83^{\dagger}$ & 4.29 & 0.34 & $\frac{6}{13}$ & $\frac{7}{13}$ & 0.79 & 0.29 \\
\hline Raspollini et al. [56] & Prognosis & $0.79^{\dagger}$ & $0.46^{\dagger}$ & 1.45 & 0.47 & $\frac{28}{52}$ & $\frac{24}{52}$ & 0.63 & 0.29 \\
\hline Hartmann et al. [57] & Prognosis & $0.86^{*}$ & $0.86^{*}$ & 6.14 & 0.16 & $\frac{21}{28}$ & $\frac{7}{28}$ & 0.95 & 0.05 \\
\hline Selvanayagam et al. [59] & Chemoresistance & $1.00^{\dagger}$ & $1.00^{\dagger}$ & $\infty$ & 0.00 & $\frac{4}{8}$ & $\frac{4}{8}$ & 1.00 & 0.00 \\
\hline Kamazawa et al. [61] & Chemoresponse & $1.00^{*}$ & $0.83^{\dagger}$ & 6.00 & 0.00 & $\frac{21}{27}$ & $\frac{5}{27}$ & 0.95 & 0.00 \\
\hline
\end{tabular}

*Value stated in reference.

$\dagger$ Value calculated.

C: condition presence.

T: test result.

RFS: Relapse Free Survival.

PFS: Progression Free Survival.

et al. [24], Schlumbrecht and Seidal[40], and Denkert et al. [45]. These models would need further investigation to determine their predictive ability. Of the papers in this group, Spentzos et al. [58] appears to have the best predictive ability when classifying patients into two clusters with significantly different survival times.

\section{Linear regression}

Two papers reported the success of model assessed using linear regression: Glaysher et al. [41] and Kang et al. [31]. These studies plotted the predicted values or model score against the measured values and applied linear regression to obtain a line of best fit. The $R^{2}$ or $R_{\text {adj }}^{2}$ of this line is then calculated to assess the discrimination of the model. Glaysher et al. [41] achieved $R^{2}=0.901\left(R_{\mathrm{adj}}^{2}=0.836\right)$ for a model predicting resistance to cisplatin via crossvalidation and Kang et al. [31] achieved $R^{2}=0.84$ for a model predicting recurrence-free survival in the data set on which it was derived. These values suggest a good level of predictive ability, both in terms of calibration and discrimination, with the model by Glaysher et al. [41] achieving the better predictions.

\section{Cox proportional hazards models}

When studies identified by this review applied the Cox proportional hazards model to predict patient outcome, it was common for the main analysis of the model to be assessing whether the gene signature was found to be significant and whether the signature was an independent predictor. However, the application of this model to an independent data set was much less common. As may be seen from Table 6, the success of many models was judged using the significance of covariates including the gene signature in the model. It is likely that this model was not applied to external data sets due to subtleties in what the model predicts when compared to methods such as linear regression. Whereas in linear regression the survival times are predicted directly, Cox proportional hazards regression predicts hazard ratios. Royston and Altman [86] developed techniques for the external validation of Cox proportional hazards models by application to an independent data set. These rely on having at least the weights of the variables included in the linear predictor, and ideally the baseline survival function. The first allows the assessment of the discriminatory power of a model, whereas the second is also required to allow the calibration of the model to be assessed. Royston and Altman [86] are of the opinion that the inclusion of a log-rank test $\mathrm{p}$-value is not informative due to the irrelevance of the null hypothesis being tested, and hence this should not be considered when judging model performance. An alternative to the log-rank test to compare survival between groups would be time-dependent ROC curves [87].

\section{Failure to predict}

Of the studies identified by this review, some models failed to achieve significant predictive ability. These include Lisowska et al. [23], Vogt et al. [62] and Brun et al. [34]. Of these papers, Vogt et al. [62] and Brun et al. [34] both considered small numbers of genes when 
Table 13 Prediction metrics for studies reporting hazard ratios

\begin{tabular}{|c|c|c|c|c|c|c|}
\hline Study & Prediction & Classes & HR & $95 \% \mathrm{Cl}$ & Median survival & $P$ value \\
\hline Jeong et al. [22] & OS & YA subgroup vs. YI subgroup & 0.5 & $0.31-0.82$ & & 0.005 \\
\hline Roque et al. [24] & OS & High vs. low TUBB3 staining & 3.66 & $1.11-12.05$ & 707 days vs. not reached & 0.03 \\
\hline Kang et al. [31] & OS & High vs. low score & 0.33 & $0.13-0.86$ & 1.8 years vs. 2.9 years & $<0.001$ \\
\hline Skirnisdottir and Seidal [35] & Recurrence & p53 -ve vs. +ve & 4.12 & $1.41-12.03$ & & 0.009 \\
\hline Schlumbrecht et al. [40] & RFS & EIG121 high vs. low & 1.13 & $1.02-1.26$ & & 0.021 \\
\hline Yoshihara et al. [43] & PFS & High vs. low score & 1.64 & $1.27-2.13$ & & 0.0001 \\
\hline Denkert et al. [45] & OS & Low vs. high score & 1.7 & $1.1-2.6$ & & 0.021 \\
\hline Crijns et. al [47] & OS & & 1.94 & $1.19-3.16$ & & 0.008 \\
\hline Netinatsunthorn et al. [51] & RFS & Yes vs. no WT1 staining & 3.36 & $1.60-7.03$ & & 0.0017 \\
\hline Spentzos et al. [54] & OS & Resistant vs. sensitive & 3.9 & $1.3-11.4$ & 41 months vs. not reached & $<0.001^{\dagger}$ \\
\hline Raspollini et al. [56] & OS & No vs. yes COX-2 staining & 0.23 & $0.06-0.77$ & & 0.017 \\
\hline Spentzos et al. [58] & OS & High vs. low score & 4.6 & $2.0-10.7$ & 30 months vs. not reached & 0.0001 \\
\hline
\end{tabular}

Calculated value.

HR: Hazard Ratio.

OS: Overall Survival.

RFS: Relapse Free Survival.

PFS: Progression Free Survival.

$\mathrm{Cl}$ : Confidence Interval.

constructing their models. It is possible then that these models failed because no informative genes were considered. Conversely, Lisowska [23] applied their modelling technique to over 47000 genes using 127 patients. It is therefore a possibility that genes were selected by their model purely by chance rather than due to true explanatory ability. This model was tested using an independent data. When the model was applied to this data set it performed poorly, suggesting that the genes chosen did not generalise to the second cohort of patients. Neither Vogt et al. [62] nor Brun et al. [34] reported measuring the precision or accuracy of the gene expression measurements. Lisowska et al. [23] used RT-PCR to measure the expression of 18 genes from the microarray, but the RTPCR measurements were carried out on a separate set of samples and hence are not useful when considering accuracy. It is therefore unknown whether the gene expression measurement techniques applied by these studies were sufficiently accurate.

\section{Discussion}

The papers identified as part of this review tackled the important issue of chemoresistance and survival prediction in ovarian cancer via gene or protein expression. The concept of identifying gene signatures is popular, but requires careful handling to extract the information required for this to be successful. It was observed that of the many different tissue preservation techniques applied, the most common were fresh-frozen and formalin fixed, paraffin embedded tissue. It is our opinion that, due to the high quality expression measurements that may now be achieved with FFPE tissue, this is the most appropriate choice for research intended to translate into a clinical setting.

It was found that the majority of the studies included in this review were heterogeneous with respect to the histological type of the patient cohort. This suggests that, due to the differing response of different types of ovarian cancer to chemotherapy, the gene signatures may be identifying different pathways and mechanisms. However, it should also be noted that although 27 of the 42 studies were heterogeneous, 12 of these consisted of greater than $80 \%$ serous samples. Therefore, for these studies the inclusion of multiple histological types is likely to have less effect on the gene signature and mechanisms highlighted could be expected to occur in serous ovarian cancer. It would be advisable for future studies to include histological type and grade as model features.

The majority of studies identified by this review attempt to classify patients into groups with different characteristics, for example 'poor prognosis' and 'good prognosis' or 'chemosensitive' and 'chemoresistant'. However, variables such as response to chemotherapy and prognosis are rarely so well separated into classes; they are by nature continuous variables. Altman and Royston [88] are clear that dichotomising continuous variables into categories (such as high-risk vs. low-risk) should be avoided, as it results in loss of information and may lead to underestimation of variation and the masking of non-linearity. Arbitrary choices of cutoff values may further obscure the situation, when the original continuous variable could 
serve the same purpose in many models. In terms of a clinical test it therefore may be more appropriate to apply alternative techniques, such as various types of regression, to obtain a real valued prediction of patient outcome.

It was noted that the metrics reported as measures of predictive ability vary between studies. These vary in the amount of information conveyed and hence care should be taken to use metrics that fully describe the model. Sensitivity and specificity are commonly reported for classification techniques and, together with the numbers of patients in each class in the data set, allows the probabilities of a patient having the condition of interest given that they have tested positive or negative. It is the ultimate aim of most classification studies to obtain these probabilities, as it allows the predictive ability of the test to be assessed and the applicability of the test to be evaluated. Of the studies reporting sensitivity, specificity and related information, the best predictive ability was achieved by Hartmann et al. [57] and the worst by Helleman et al. [53]. It is important to note that from the sensitivity and specificity the model by Helleman et al. [53] does not appear to be any worse than some of the others, but these probabilities incorporate the prevalence of the condition of interest in the test population. It would therefore be highly informative to recalculate these probabilities using the prevalence of the condition in the population of ovarian cancer patients. Since some of the test populations were not representative of the overall population (having so called 'spectrum bias'), this would give a much more reliable indication of the predictive ability of the models in a clinical setting.

One of the main aims of the studies identified was to obtain a 'gene signature', the expression of which can explain and predict the response in the patient. To this end, the majority of the papers (32 of 42) provided full or partial list of the genes selected by the modelling process. An analysis of these gene signatures resulted in the conclusion that the signatures were very dissimilar, with the most commonly selected gene appearing in only four papers. $93.53 \%$ of genes were selected by only one paper. This seems to indicate that the gene signatures identified were not based on underlying cellular processes, or at least that the processes being highlighted were not the same across the papers. It should be noted that many of the studies used cohorts of patients who were heterogeneous in terms of chemotherapy treatment and, due to the development of resistance to chemotherapy via gene expression changes, this may affect the genes found to be explanatory. It may be that several gene signatures from sub-populations of patients treated with different drugs are combining and hence reducing the predictive ability of the models.
In order to assess the biological relevance of the genes selected for the gene signatures, gene set enrichment analysis was carried out. This technique is used to highlight processes and pathways that are over-represented in the gene signature compared to the set of all genes. For the purposes of this review, two groups of studies were considered: those where the patients were treated with platinum and taxane, and those where the patients were treated with other platinum based treatments. These groups were selected due to the low numbers of studies using a single treatment option. For example, there were no studies considering platinum, taxane or cyclophosphamide as single agents. Following the analysis, 30 KEGG terms were returned for each group. Of these, each list comprised of approximately half cancer related terms. Of these the majority were processes often up- or down-regulated in cancer cells, such as proliferation, apoptosis, and motility and metastasis [89]. It is unclear whether the change in regulation of these processes is further altered in response to specific chemotherapy treatments. However, one process worthy of additional consideration is DNA repair. DNA repair is known to be an important mechanism in cancer both though cancer development when downregulated or mutated [75] and resistance to DNA damaging chemotherapy when up-regulated [76]. Therefore, the strong presence of DNA repair terms may suggest the presence of platinum resistance pathways in the gene signatures. It is the authors' opinion that, although the combined gene signatures appear not to include predictive chemotherapy-specific information, they may be capable of providing prognostic information. It is also thought that some studies, such as Glaysher et al., may include genes relevant to additional chemotherapy-specific processes which are 'drowned out' when combined with other signatures.

\section{Conclusion}

It is clear that the prediction of response to chemotherapy in ovarian cancer is an ongoing research problem that has been attracting attention for many years. However, although many studies have been published, a clinical tool is still not available. It is our belief that, although not yet accomplished, progress within the field suggests that the development of a predictive model is possible. There is great variability between the approaches and success of existing studies in the literature, and there have been very high levels of variation in the genes identified as explanatory. It is the authors' opinion that, if more care is taken when selecting the patients for inclusion to control for treatment history, these gene signatures may be simplified and models able to predict response to treatment may be developed. 


\section{Additional files}

\section{Additional file 1: PubMed search terms. \\ Additional file 2: PRISMA Checklist. \\ Additional file 3: Bias assessment, including QUADAS-2 and CEBM levels of evidence.}

\section{Competing interests}

The authors declare that they have no competing interests.

\section{Authors' contributions}

IAC and RSS conceived and planned the study. Literature searches were carried out by KLL. KLL drafted the paper, which was critically reviewed and revised by IAC and RSS. All authors read and approved the final manuscript.

\section{Acknowledgements}

KLL acknowledges support from an EPSRC PhD studentship (though MOAC DTC, EP/F500378/1). RSS acknowledges support from an MRC Biostatistics Fellowship.

\section{Author details}

${ }^{1}$ MOAC DTC, University of Warwick, Gibbet Hill Road, CV4 7AL, Coventry, UK. ${ }^{2}$ Warwick Medical School, University of Warwick, Gibbet Hill Road, CV4 7AL, Coventry, UK. ${ }^{3}$ Systems Biology Centre, University of Warwick, Gibbet Hill Road, CV4 7AL, Coventry, UK.

Received: 8 August 2014 Accepted: 20 February 2015

Published online: 11 March 2015

\section{References}

1. Office for National Statistics: Cancer Incidence and Mortality in the United Kingdom. 2008-2010. http://www.ons.gov.uk/ons/rel/cancer-unit/ cancer-incidence-and-mortality/2008-2010/rft-cancer-incidence-andmortality-in-the-uk--2008-2010.xls.

2. Office for National Statistics: Cancer Survival Rates, Cancer Survival in England, Patients Diagnosed 2005-2009 and Followed up to 2010. http:// www.ons.gov.uk/ons/rel/cancer-unit/cancer-survival-rates/2005-2009-followed-up-to-2010/tbl-cancer-survival.xls.

3. Li M, Yin J, Mao N, Pan L. Upregulation of phosphorylated cofilin 1 correlates with taxol resistance in human ovarian cancer in vitro and in vivo. Oncology Rep. 2013;29(1):58-66.

4. Rubin SC, Randall TC, Armstrong KA, Chi DS, Hoskins WJ. Ten-year follow-up of ovarian cancer patients after second-look laparotomy with negative findings. Obstet Gynecol. 1999;93(1):21-4

5. Armstrong DK. Relapsed ovarian cancer: challenges and management strategies for a chronic disease. The Oncologist. 2002;7(Supplement 5): 20-8.

6. Di Nicolantonio F, Mercer SJ, Knight LA, Gabriel FG, Whitehouse PA, Sharma S, et al. Cancer cell adaptation to chemotherapy. BMC Cancer. 2005;5(1):78.

7. National Institute for Health and Care Excellence: Guidance on the use of paclitaxel in the treatment of ovarian cancer. http://www.nice.org.uk/ guidance/ta55.

8. Goff BA. Advanced ovarian cancer: what should be the standard of care? J Gynecol Oncol. 2013;24(1):83-91.

9. Winter WE, Maxwell GL, Tian C, Carlson JW, Ozols RF, Rose PG, et al. Prognostic factors for stage iii epithelial ovarian cancer: a gynecologic oncology group study. J Clin Oncol. 2007;25(24):3621-7.

10. Cancer Research UK: Ten-year survival over time. http://www. cancerresearchuk.org/prod_consump/groups/cr_common/@nre/@sta/ documents/generalcontent/surv_10yrtrends_selcancers_xls.xls.

11. Slodkowska EA, Ross JS. Mammaprint ${ }^{\mathrm{TM}} 70$-gene signature: another milestone in personalized medical care for breast cancer patients. Expert Rev Mol Diagn. 2009;9(5):417-22.

12. Oncotype DX : Underlying Technology. http://breast-cancer.oncotypedx. com/en-US/Professional-Invasive/WhatIsTheOncotypeDXBreastCancer Test/UnderlyingTechnology.aspx.

13. Oncotype DX : What Is the Colon cancer test? http://colon-cancer. oncotypedx.com/en-US/Professional/WhatlsTheColonCancerTest.aspx.
14. Oncotype DX ${ }^{\circ}$ : Development. http://prostate-cancer.oncotypedx.com/ en-US/Professional/IntroducingGPS/Development.aspx.

15. Efron B. The efficiency of cox's likelihood function for censored data. J Am Stat Assoc. 1977;72(359):557-65

16. Gillet J-P, Calcagno AM, Varma S, Marino M, Green LJ, Vora MI, et al. Redefining the relevance of established cancer cell lines to the study of mechanisms of clinical anti-cancer drug resistance. Proc Nat Acad Sci. 2011;108(46):18708-13.

17. Cree IA, Glaysher S, Harvey AL. Efficacy of anti-cancer agents in cell lines versus human primary tumour tissue. Curr Opin Pharmacol. 2010;10(4): 375-9.

18. Whiting PF, Rutjes AW, Westwood ME, Mallett S, Deeks JJ, Reitsma JB, et al. Quadas-2: a revised tool for the quality assessment of diagnostic accuracy studies. Ann Intern Med. 2011;155(8):529-36.

19. The Oxford 2011 Levels of Evidence (OCEBM Levels of Evidence Working Group). http://www.cebm.net/index.aspx?o=5653.

20. Wang X, Terfve C, Rose JC, Markowetz F. Htsanalyzer: an r/bioconductor package for integrated network analysis of high-throughput screens. Bioinformatics. 2011;27(6):879.

21. Benjamini Y, Hochberg Y. Controlling the false discovery rate: a practical and powerful approach to multiple testing. Journal of the Royal Statistical Society. Series B (Methodological). 1995:57(1):289-300.

22. Jeong W, Kim S-B, Sohn BH, Park Y-Y, PARK ES, Kim SC, et al. Activation of yap1 is associated with poor prognosis and response to taxanes in ovarian cancer. Anticancer Res. 2014;34(2):811-7.

23. Lisowska KM, Olbryt M, Dudaladava V, Pamula-Pilat J, Kujawa K, Grzybowska E, et al. Gene expression analysis in ovarian cancer - faults and hints from dna microarray study. Front Oncol. 2014;4:6.

24. Roque DM, Buza N, Glasgow M, Bellone S, Bortolomai I, Gasparrini S, et al. Class iii beta-tubulin overexpression within the tumor microenvironment is a prognostic biomarker for poor overall survival in ovarian cancer patients treated with neoadjuvant carboplatin/paclitaxel. Clin Exp Metastasis. 2014;31(1):101-10.

25. Schwede M, Spentzos D, Bentink S, Hofmann O, Haibe-Kains B, Harrington D, et al. Stem cell-like gene expression in ovarian cancer predicts type ii subtype and prognosis. PLoS One. 2013;8:57799.

26. Verhaak RG, Tamayo P, Yang JY, Hubbard D, Zhang H, Creighton CJ, et al. Prognostically relevant gene signatures of high-grade serous ovarian carcinoma. J Clin Invest. 2013;123(1):517-25.

27. Obermayr E, Castillo-Tong DC, Pils D, Speiser P, Braicu I, Van Gorp T, et al. Molecular characterization of circulating tumor cells in patients with ovarian cancer improves their prognostic significance - a study of the ovcad consortium. Gynecol Oncol. 2013;128(1):15-21

28. Han Y, Huang H, Xiao Z, Zhang W, Cao Y, Qu L, et al. Integrated analysis of gene expression profiles associated with response of platinum/paclitaxel-based treatment in epithelial ovarian cancer. PLoS One. 2012;7:52745.

29. Hsu FH, Serpedin E, Hsiao TH, Bishop AJ, Dougherty ER, Chen Y. Reducing confounding and suppression effects in tcga data: an integrated analysis of chemotherapy response in ovarian cancer. BMC Genomics. 2012;13 Suppl 6:13

30. Liu Y, Sun Y, Broaddus R, Liu J, Sood AK, Shmulevich I, et al. Integrated analysis of gene expression and tumor nuclear image profiles associated with chemotherapy response in serous ovarian carcinoma. PLoS One. 2012;7:36383

31. Kang J, D'Andrea AD, Kozono D. A dna repair pathway-focused score for prediction of outcomes in ovarian cancer treated with platinum-based chemotherapy. J Natl Cancer Inst. 2012;104:670-81.

32. Gillet JP, Calcagno AM, Varma S, Davidson B, Bunkholt Elstrand M Ganapathi R, et al. Multidrug resistance-linked gene signature predicts overall survival of patients with primary ovarian serous carcinoma. Clin Cancer Res. 2012;18:3197-206.

33. Ferriss JS, Kim Y, Duska L, Birrer M, Levine DA, Moskaluk C, et al. Multi-gene expression predictors of single drug responses to adjuvant chemotherapy in ovarian carcinoma: predicting platinum resistance. PLoS One. 2012;7:30550.

34. Brun JL, Cortez A, Lesieur B, Uzan S, Rouzier R, Darai E. Expression of $m m p-2,-7,-9, m t 1-m m p$ and timp-1 and -2 has no prognostic relevance in patients with advanced epithelial ovarian cancer. Oncol Rep. 2012:27(4):1049-57

35. Skirnisdottir I, Seidal T. The apoptosis regulators p53, bax and puma: Relationship and impact on outcome in early stage (figo i-ii) ovarian 
carcinoma after post-surgical taxane-based treatment. Oncol Rep. 2012;27(3):741-7

36. Brenne K, Nymoen DA, Hetland TE, Trope' CG, Davidson B. Expression of the ets transcription factor ehf in serous ovarian carcinoma effusions is a marker of poor survival. Hum Pathol. 2012;43(4):496-505.

37. Sabatier R, Finetti P, Bonensea J, Jacquemier J, Adelaide J, Lambaudie E, et al. A seven-gene prognostic model for platinum-treated ovarian carcinomas. Br J Cancer. 2011;105:304-11.

38. Gillet JP, Wang J, Calcagno AM, Green LJ, Varma S, Bunkholt Elstrand M, et al. Clinical relevance of multidrug resistance gene expression in ovarian serous carcinoma effusions. Mol Pharm. 2011;8(6):2080-8.

39. Chao SY, Chiang JH, Huang AM, Chang WS. An integrative approach to identifying cancer chemoresistance-associated pathways. BMC Med Genomics. 2011;4:23.

40. Schlumbrecht MP, Xie SS, Shipley GL, Urbauer DL, Broaddus RR. Molecular clustering based on eralpha and eig121 predicts survival in high-grade serous carcinoma of the ovary/peritoneum. Mod Pathol. 2011;24:453-62.

41. Glaysher S, Gabriel FG, Johnson P, Polak M, Knight LA, Parker K, et al. Molecular basis of chemosensitivity of platinum pre-treated ovarian cancer to chemotherapy. Br J Cancer. 2010;103:656-62.

42. Yan X, Yin J, Yao H, Mao N, Yang Y, Pan L. Increased expression of annexin a3 is a mechanism of platinum resistance in ovarian cancer Cancer Res. 2010;70:1616-24.

43. Yoshihara K, Tajima A, Yahata T, Kodama S, Fujiwara H, Suzuki M, et al. Gene expression profile for predicting survival in advanced-stage serous ovarian cancer across two independent datasets. PLoS One. 2010;5(3): 9615.

44. Williams PD, Cheon S, Havaleshko DM, Jeong H, Cheng F, Theodorescu $\mathrm{D}$, et al. Concordant gene expression signatures predict clinical outcomes of cancer patients undergoing systemic therapy. Cancer Res. 2009;69: 8302-9.

45. Denkert C, Budczies J, Darb-Esfahani S, Gyorffy B, Sehouli J, Konsgen D, et al. A prognostic gene expression index in ovarian cancer - validation across different independent data sets. J Pathol. 2009;218(2):273-80.

46. Matsumura N, Huang Z, Baba T, Lee PS, Barnett JC, Mori S, et al. Yin yang 1 modulates taxane response in epithelial ovarian cancer. Mol Cancer Res. 2009;7:210-20.

47. Crijns AP, Fehrmann RS, de Jong S, Gerbens F, Meersma GJ, Klip HG, et al. Survival-related profile, pathways, and transcription factors in ovarian cancer. PLoS Med. 2009;6:24.

48. Mendiola M, Barriuso J, Redondo A, Marino-Enriquez A, Madero R, Espinosa E, et al. Angiogenesis-related gene expression profile with independent prognostic value in advanced ovarian carcinoma. PLoS One. 2008;3(12):4051.

49. Gevaert O, De Smet F, Van Gorp T, Pochet N, Engelen K, Amant F, et al. Expression profiling to predict the clinical behaviour of ovarian cancer fails independent evaluation. BMC Cancer. 2008;8:18.

50. Bachvarov D, L'Esperance S, Popa I, Bachvarova M, Plante M, Tetu B. Gene expression patterns of chemoresistant and chemosensitive serous epithelial ovarian tumors with possible predictive value in response to initial chemotherapy. Int J Oncol. 2006;29(4):919-33.

51. Netinatsunthorn W, Hanprasertpong J, Dechsukhum C, Leetanaporn R, Geater A. Wt1 gene expression as a prognostic marker in advanced serous epithelial ovarian carcinoma: an immunohistochemical study. BMC Cancer. 2006;6:90.

52. De Smet F, Pochet NL, Engelen K, Van Gorp T, Van Hummelen P, Marchal K, et al. Predicting the clinical behavior of ovarian cancer from gene expression profiles. Int J Gynecol Cancer. 2006;16 Suppl 1:147-51.

53. Helleman J, Jansen MP, Span PN, van Staveren IL, Massuger LF, Meijer-van Gelder ME, et al. Molecular profiling of platinum resistant ovarian cancer. Int J Cancer. 2006;118(8):1963-71.

54. Spentzos D, Levine DA, Kolia S, Otu H, Boyd J, Libermann TA, et al. Unique gene expression profile based on pathologic response in epithelial ovarian cancer. J Clin Oncol. 2005;23:7911-8.

55. Jazaeri AA, Awtrey CS, Chandramouli GV, Chuang YE, Khan J, Sotiriou C, et al. Gene expression profiles associated with response to chemotherapy in epithelial ovarian cancers. Clin Cancer Res. 2005;11:6300-10.

56. Raspollini MR, Amunni G, Villanucci A, Boddi V, Taddei GL. Increased cyclooxygenase-2 (cox-2) and p-glycoprotein-170 (mdr1) expression is associated with chemotherapy resistance and poor prognosis. analysis in ovarian carcinoma patients with low and high survival. Int J Gynecol Cancer. 2005;15:255-60.

57. Hartmann LC, Lu KH, Linette GP, Cliby WA, Kalli KR, Gershenson D, et al. Gene expression profiles predict early relapse in ovarian cancer after platinum-paclitaxel chemotherapy. Clin Cancer Res. 2005;11:2149-55.

58. Spentzos D, Levine DA, Ramoni MF, Joseph M, Gu X, Boyd J, et al. Gene expression signature with independent prognostic significance in epithelial ovarian cancer. J Clin Oncol. 2004;22:4700-10.

59. Selvanayagam ZE, Cheung TH, Wei N, Vittal R, Lo KW, Yeo W, et al. Prediction of chemotherapeutic response in ovarian cancer with dna microarray expression profiling. Cancer Genet Cytogenet. 2004;154:63-6.

60. Iba T, Kigawa J, Kanamori Y, Itamochi H, Oishi T, Simada M, et al. Expression of the c-myc gene as a predictor of chemotherapy response and a prognostic factor in patients with ovarian cancer. Cancer Sci. 2004;95(5):418-23.

61. Kamazawa S, Kigawa J, Kanamori Y, Itamochi H, Sato S, Iba T, et al. Multidrug resistance gene- 1 is a useful predictor of paclitaxel-based chemotherapy for patients with ovarian cancer. Gynecol Oncol. 2002;86: 171-6.

62. Vogt U, Falkiewicz B, Bielawski K, Bosse U, Schlotter CM. Relationship of c-myc and erbb oncogene family gene aberrations and other selected factors to ex vivo chemosensitivity of ovarian cancer in the modified atp-chemosensitivity assay. Acta Biochim Pol. 2000;47(1):157-64.

63. Medeiros F, Rigl CT, Anderson GG, Becker SH, Halling KC. Tissue handling for genome-wide expression analysis: a review of the issues, evidence, and opportunities. Arch Pathol Lab Med. 2007;131(12):1805-16.

64. Rentoft M, Coates PJ, Laurell G, Nylander K. Transcriptional profiling of formalin fixed paraffin embedded tissue: pitfalls and recommendations for identifying biologically relevant changes. PloS One. 2012;7(4):35276.

65. Takano M, Tsuda H, Sugiyama T. Clear cell carcinoma of the ovary: is there a role of histology-specific treatment. J Exp Clin Cancer Res. 2012;31(53):35.

66. Hess V, A'Hern R, Nasiri N, King DM, Blake PR, Barton DP, et al. Mucinous epithelial ovarian cancer: a separate entity requiring specific treatment. J Clin Oncol. 2004;22(6):1040-4.

67. Oldenhuis C, Oosting S, Gietema J, De Vries E. Prognostic versus predictive value of biomarkers in oncology. Eur J Cancer. 2008;44(7): 946-53.

68. Tezak Z, Kondratovich MV, Mansfield E. Us fda and personalized medicine: in vitro diagnostic regulatory perspective. Personalized Med. 2010;7(5):517-30.

69. Tapia G, Diaz-Padilla I. Molecular mechanisms of platinum resistance in ovarian cancer In: Diaz-Padilla I, editor. Ovarian Cancer - A Clinical and Translational Update. InTech; 2013.

70. Citri A, Yarden Y. Egf-erbb signalling: towards the systems level. Nat Rev Mol Cell Biol. 2006;7(7):505-16.

71. Tan F, Thiele CJ, Zhijie L. Neurotrophin signaling in cancer In: Kostrzewa RM, editor. Handbook of Neurotoxicity. New York: Springer; 2014.

72. McLean GW, Carragher NO, Avizienyte E, Evans J, Brunton VG, Frame MC. The role of focal-adhesion kinase in cancer-a new therapeutic opportunity. Nat Rev Cancer. 2005;5(7):505-15.

73. Elliott $\mathrm{RL}$, Blobe $\mathrm{GC}$. Role of transforming growth factor beta in human cancer. J Clin Oncol. 2005;23(9):2078-93.

74. Levine AJ, Bargonetti J, Bond GL, Hoh J, Onel K, Overholtzer M, et al. The p53 network In: Zambetti GP, editor. The P53 Tumor Suppressor Pathway and Cancer. US: Springer; 2005.

75. Neumann AS, Sturgis EM, Wei Q. Nucleotide excision repair as a marker for susceptibility to tobacco-related cancers: A review of molecular epidemiological studies. Mol Carcinog. 2005;42(2):65-92.

76. Martin LP, Hamilton TC, Schilder RJ. Platinum resistance: the role of dna repair pathways. Clin Cancer Res. 2008;14(5):1291-5.

77. Galletti E, Magnani M, Renzulli ML, Botta M. Paclitaxel and docetaxel resistance: molecular mechanisms and development of new generation taxanes. ChemMedChem. 2007;2(7):920-42.

78. Jekunen A, Christen R, Shalinsky D, Howell S. Synergistic interaction between cisplatin and taxol in human ovarian carcinoma cells in vitro. British J Cancer. 1994;69(2):299.

79. Hall A, Tilby M. Mechanisms of action of, and modes of resistance to, alkylating agents used in the treatment of haematological malignancies. Blood Rev. 1992;6(3):163-73. 
80. Pópulo H, Lopes JM, Soares P. The mtor signalling pathway in human cancer. Int J Mol Sci. 2012;13(2):1886-918.

81. Dhillon A, Hagan S, Rath O, Kolch W. Map kinase signalling pathways in cancer. Oncogene. 2007;26(22):3279-90.

82. Hembruff SL, Cheng N. Chemokine signaling in cancer: Implications on the tumor microenvironment and therapeutic targeting. Cancer Ther. 2009; 7 (A):254.

83. Kowanetz M, Ferrara N. Vascular endothelial growth factor signaling pathways: therapeutic perspective. Clinical Cancer Res. 2006;12(17): 5018-22.

84. Cree IA. Designing personalised cancer treatments. J Control Release. 2013;172(2):405-9.

85. Bernstein C, Prasad AR, Nfonsam V, Bernstein H. Dna damage, dna repair and cancer In: Chen C, editor. New Research Directions in DNA Repair. InTech; 2013.

86. Royston P, Altman DG. External validation of a cox prognostic model: principles and methods. BMC Med Res Methodol. 2013;13(1):33.

87. Heagerty PJ, Lumley T, Pepe MS. Time-dependent roc curves for censored survival data and a diagnostic marker. Biometrics. 2000;56(2):337-44.

88. Altman DG, Royston P. Statistics notes: the cost of dichotomising continuous variables. BMJ: British Med J. 2006;332(7549):1080.

89. Hanahan D, Weinberg RA. The hallmarks of cancer. Cell. 2000;100(1): $57-70$.

Submit your next manuscript to BioMed Central and take full advantage of:

- Convenient online submission

- Thorough peer review

- No space constraints or color figure charges

- Immediate publication on acceptance

- Inclusion in PubMed, CAS, Scopus and Google Scholar

- Research which is freely available for redistribution

Submit your manuscript at www.biomedcentral.com/submit

\section{Biomed Central}

